\title{
A fast and versatile method for spectral emissivity measurement at high temperatures
}

\author{
Abdelmagid El Bakali ${ }^{{ }^{*}}$, Rémi Gilblas ${ }^{1}$, Thomas Pottier ${ }^{1}$, Yannick Le Maoult ${ }^{1}$ \\ (1) Institut Clément Ader (ICA) ; Université de Toulouse ; CNRS, IMT Mines Albi, INSA, ISAE-SUPAERO, \\ UPS ; Campus Jarlard, F-81013 Albi, France \\ 1* corresponding author: aelbakal@mines-albi.fr
}

Keywords: Spectral emissivity, Total emissivity, Platinum, IR spectrometry, IR thermography, High temperature, Direct radiometric methods, IR heating, Elliptical furnace

\begin{abstract}
In this paper, the development of a new device for high temperature emissivity measurement is described. This device aims at measuring both spectral and total emissivity for a thermal range of 600 to $1000^{\circ} \mathrm{C}$. The device main targeted properties were both versatility and simplicity. To do so, a rigorous selection was made for each component: heating system, heat source, sample holder, measuring devices. Sample dimensions and the corresponding sample holder were optimized through a ray tracing model computation. Selection of sensors to compute a total emissivity was also especially discussed. A NIR spectrometer, and two MIR cameras covering [3-5] and [7.5-13] $\mu \mathrm{m}$ equipped with optical filters were chosen for spectral measurements. The major impediment was the separation between sample signal and various spurious signals emitted by environment. A specific measurement methodology was then made for each bandwidth to resolve this issue. A reference material was chosen for the device validation: Platinum. Spectral emissivity measurements were then compared to values from a commercial spectrometer. A good agreement was found for NIR and the first MIR band measurements and a higher error was seen in MIR second band which is explained by a less favorable signal to noise ratio. Integrated emissivity is then calculated and compared to values found in the literature. A good agreement between those values is found and similar trends with temperature are observed. The device is then validated for spectral and total emissivity measurement. Device versatility and simplicity allows an easy adaptation to a large area of applications.
\end{abstract}




\section{Introduction}

Development of numerical simulation and radiative thermometry in recent years has led to an increasing need for emissivity experimental assessment. Emissivity is a complex property that can be declined in directional spectral emissivity, directional total emissivity, spectral hemispherical emissivity or total hemispherical emissivity [1]. In practice, total hemispherical and spectral directional emissivities are the most needed properties. Total hemispherical emissivity is generally used as a boundary condition for classical heat transfer computation or in surface to surface radiative models. It is therefore the most common and spread out value of emissivity. It is usually determined with devices like thermopile or bolometers [2]... On the other hand, spectral directional emissivity is useful for infrared temperature measurements with devices (IR pyrometer, IR camera...) operating within a specific bandwidth [3]. Indeed, spectral emissivity may exhibit significant variations with the wavelength [4] and having the corresponding spectral emissivity is compulsory in assessing the measurement metric.

Emissivity depends on numerous parameters such as material roughness, chemical composition, manufacturing process, surface oxidation, temperature ([5], [6]). Emissivity values found in literature usually lack such information about the sample used for the measurement. This uncertainty on emissivity can be problematic for applications where accuracy is required (thermoforming, hot stamping...). Commercial devices can generally be used up to $400^{\circ} \mathrm{C}$ for spectral emissivity which is particularly suitable for thermoplastic and thermoplastic composite materials. However, it is insufficient for high temperature metallic materials applications such as hot forming [7], superplastic forming [8], hot stamping [9]... Another issue for metallic materials is the impact of oxidation at high temperature which strongly modifies emissivity value [6] during the process. In order to have realistic heat transfer calculation or IR measurements for those processes, corresponding materials emissivity have to be measured in the same experimental conditions (temperature, atmosphere, heating rate...). Accordingly, the present paper details the development of a novel apparatus which enables emissivity measurement at high temperatures. Thermal range was guided by usual metal temperature forming range of 600 to $1100^{\circ} \mathrm{C}$ in aforementioned processes. Bandwidth was selected to match with corresponding bandwidths of most IR detectors[10]: NIR band I (BI): [0.9-2] $\mu \mathrm{m}, \mathrm{MIR}$ Band II (BII): [2.5-5] $\mu \mathrm{m}$, and MIR Band III (BIII): [7.5-13] $\mu \mathrm{m}$. Furthermore, an integrated emissivity close to total emissivity can be calculated from these bandwidths. Indeed, for a black body heated at $600^{\circ} \mathrm{C}$, Wien's displacement law enables to calculate the maximum emission wavelength:

$$
\lambda_{\max }=2898 / T(K)
$$

Then, $96 \%$ of the radiation is emitted from $0.5 \lambda_{\max }$ to $5 \lambda_{\max }$. For the lowest temperature of our requirements $\left(600^{\circ} \mathrm{C}\right)$, the radiation is then emitted between 1.65 and $16.5 \mu \mathrm{m}$. For the highest temperature $\left(1100^{\circ} \mathrm{C}\right)$, the spectral range is [1.06-10.6] $\mu \mathrm{m}$. From those calculations, the requirements of the measurement can be built.

\begin{tabular}{|c|c|c|}
\hline Temperature range & Bandwidth & atmosphere \\
\hline$[600 ; 1100]^{\circ} \mathrm{C}$ & {$[1.1 ; 16.5] \mu \mathrm{m}$} & Air, vacuum, argon \\
\hline
\end{tabular}

Table 1 : Device requirements

The main targeted properties for this device are both versatility and simplicity. In one hand, versatility opens a wide range of applications, like solar, spatial, forming applications, temperature measurements or numerical simulation. Therefore, heating and detection systems have to be flexible 
enough to perform both spectral ant total emissivity measurements while being able to easily modify heating and/or atmospheric conditions. On the other hand, simplicity is wanted to have a standalone device with a good cost-efficiency, which can be successfully implemented in non-specialist operators.

This paper is organized as follows: the first part exposes the state of the art of emissivity measurement methods. A comparison between those methods is then proposed in order to select the most suitable method. The second part describes the device development based on the method selected in the first part. Device elements are optimized to match the temperature requirements presented in Table 1. In the third part, the selection of the different sensors is detailed to comply bandwidth requirements presented in Table 1 . Those sensors are then calibrated with a blackbody for the emissivity measurement. In the fourth part, an emissivity measurement is performed on a reference material (pure Platinum).

\section{State of art and choice of the measurement method}

In this section, most of the common methods dedicated to emissivity measurement are presented in a non-exhaustive list of devices. A comparison is then made between those methods to select the most suited for hot forming applications.

\subsection{Theoretical basis}

Thermal radiation is emitted by every object with a temperature higher than absolute zero. This radiation is represented theoretically by the radiance that is expressed by the cross product of surface emissivity material and radiance emitted by a blackbody [11]:

$$
L_{\lambda}(\lambda, \theta, \varphi, T)=\varepsilon(\lambda, \theta, \varphi, T) * L^{0}(\lambda, \theta, \varphi, T)
$$

Where $L_{\lambda}$ is the monochromatic radiance and $\varepsilon$ is the surface emissivity. Radiance is defined as the emitted flux per surface and solid angle. Those parameters depend on wavelength, direction and temperature. Emissivity can then be defined as the ratio between the radiance emitted by the sample and the radiance emitted by the blackbody at the same temperature [11].

$$
\varepsilon(\lambda, \theta, \varphi, T)=\frac{L_{\lambda}(\lambda, \theta, \varphi, T)}{L^{0}(\lambda, \theta, \varphi, T)}
$$

This parameter known as directional spectral emissivity can be integrated in space to obtain a spectral hemispherical emissivity (Eq.4). Recalling that a blackbody emission is, by definition, isotropic, the (Eq.4) can then be simplified as follows (Eq.5).

$$
\begin{gathered}
\varepsilon(\lambda, T)=\frac{\int_{0}^{\pi / 2} \int_{0}^{2 \pi} \varepsilon(\lambda, \theta, \varphi, T) * L^{0}(\lambda, \theta, \varphi, T) * \cos \theta * \sin \theta * d \theta * d \varphi}{\int_{0}^{\pi / 2} \int_{0}^{2 \pi} L^{0}(\lambda, \theta, \varphi, T) * d \theta * \cos \theta * \sin \theta * d \varphi} \\
\varepsilon(\lambda, T)=\frac{\int_{0}^{\pi / 2} \int_{0}^{2 \pi} \varepsilon(\lambda, \theta, \varphi, T) * L^{0}(\lambda, T) * \cos \theta * \sin \theta * d \theta * d \varphi}{\pi * L^{0}(\lambda, T)}
\end{gathered}
$$

Directional spectral emissivity can also be integrated over wavelength to obtain a directional total emissivity (Eq.6). This latter relation (Eq.6) can then be simplified by integrating blackbody radiance over all wavelengths (Eq.7) [10]. 


$$
\begin{aligned}
& \varepsilon(\theta, \varphi, T)=\frac{\int_{0}^{\infty} \varepsilon(\lambda, \theta, \varphi, T) * L^{0}(\lambda, T) * d \lambda}{\int_{0}^{\infty} L^{0}(\lambda, T) * d \lambda} \\
& \varepsilon(\theta, \varphi, T)=\frac{\int_{0}^{\infty} \varepsilon(\lambda, \theta, \varphi, T) * L^{0}(\lambda, T) * d \lambda}{\sigma T^{4} / \pi}
\end{aligned}
$$

Finally the total hemispherical emissivity can be obtained by integrating over both wavelength and direction (Eq.8). This equation can be simplified by using (Eq.4) aiming to only have integration over wavelength (Eq.9).

$$
\begin{gathered}
\varepsilon(T)=\frac{\int_{0}^{\pi / 2} \int_{0}^{2 \pi} \int_{0}^{\infty} \varepsilon(\lambda, \theta, \varphi, T) * L^{0}(\lambda, T) * \cos \theta * \sin \theta * d \theta * d \varphi * d \lambda}{\int_{0}^{\pi / 2} \int_{0}^{2 \pi} \int_{0}^{\infty} L^{0}(\lambda, T) * \cos \theta * \sin \theta * d \theta * d \varphi * d \lambda} \\
\varepsilon(T)=\frac{\int_{0}^{\infty} \varepsilon(\lambda, T) * L^{0}(\lambda, T) * d \lambda}{\sigma T^{4} / \pi}
\end{gathered}
$$

As explained above, the total hemispherical emissivity $\varepsilon(T)$ and the spectral directional emissivity $\varepsilon(\lambda, T, \theta, \varphi)$ are the most sought values especially for high temperatures (numerical simulation and IR measurement). For both quantities, a high accuracy on the sample temperature is needed, even more for metallic materials whose emissivity is often varying with temperature. Several methods exist to determine these emissivities and will be described in the following. They can primarily be sorted in two main categories: i) the so-called direct methods, based on the emissivity definition which is directly calculated from the ratio of the radiance of a sample and the blackbody's one at the same temperature and ii) the indirect methods, assessing emissivity from the spectral reflectance of an opaque material by an application of Kirchhoff's law(Eq.10) which, in this case, reads:

$$
\begin{gathered}
\varepsilon(\lambda, T, \theta, \varphi)=1-\rho(\lambda, T, \Omega) \\
\text { With } \Omega=\iint_{S} \sin \theta \cdot d \theta . d \varphi
\end{gathered}
$$

Both direct and indirect methods provide spectral directional emissivity. It is then possible to determine a total hemispherical emissivity from spectral and spatial integration (if the bandwidth is wide enough for a given temperature).

\subsection{Emissivity measurement methods}

\subsubsection{Indirect methods}

In such approaches, the emissivity is generally assessed from reflectivity (see Eq. 10).

For spectral directional emissivity, surface reflectance $\rho(\lambda, T, \Omega)$ can be measured by various techniques such as FT-IR spectrometry equipped with an integrating sphere [12]. A high reflecting coating is applied on the internal surface of the sphere. An incident beam is directed on the sample and the following reflected flux by the sample in every direction is reflected by the sphere until it reaches the detector.

Alternatively, collecting the reflected flux all over the hemisphere can be performed by a set of mirrors (as high as 20), as in the spectrophotometry technique developed by Makino and Wakabayashi [13] [14]. This system can provide a measurement of directional spectral reflection for a bandwidth between 0.3 and $11 \mu \mathrm{m}$. This technique allows a fast measurement over a wide range of wavelength but is limited by the directional nature of the measure and also by the complexity of the optical paths required to redirect the reflected flux (mirrors and diffraction gratings). Reflectance can 
also be measured from mirror-based techniques. In such configuration, the sample and the detector are both placed inside a hemispherical mirror. The sample then reflects an incoming light beam that passes through a hole in the mirror. The detector thus collects the reflected heat flux from every direction and provides a measurement of the directional hemispherical reflectivity. In such approach, only one incidence angle can be investigated at a time. Later works have proven possible the evolution of this technique for varying incidence angles [15]. In this latter paper, Hameury et al. have used serval holes in the mirror to reach 5 different angles of incidence ranging from $12^{\circ}$ to $60^{\circ}$. Nevertheless, in such configuration, optical paths become complex and requires many optical components and the system ultimately loses the inherent simplicity of mirror-based devices.

An alternative method developed by Fu and al. is based on the illumination of a sample by $6150 \mathrm{~mm}$ long quartz lamps. Located at the periphery of a hemisphere, these conditions lead to the measurement of reflectance, directly linked with emissivity as denoted by equation 10. Performing the measurement at 10 wavelengths with a spectrometer, the method enables to determine on-line the temperature, the emissivity and the lamp radiance for each wavelength. This method is inherently few sensitive to temperature knowledge but is valid only for diffuse surfaces.

Another active indirect method is thermoreflectometry[16][17]. The aim of this method is to measure an object true temperature from thermography with a direct correction of the emissivity at two wavelengths. This latter correction being achieved from the reflection of two laser beams illuminating the sample at 1.3 and $1.55 \mu \mathrm{m}$. The advantage of this technique is the time-continuous nature of the measurement of emissivity at high temperatures. The constraints are the need of flat and opaque sample and the fact that emissivity measurement is limited to two wavelengths.

Total directional emissivity can be obtained by numerical integration of directional hemispherical reflectivity presented before, but also by operating the reflectivity measurement with a very wide band source (heated carbide ring) and detector (pyroelectric, bolometric and/or thermopile[19]). The challenge is here to set the spectral bandwidth to ensure that all the reflected flux is detected for every wavelength.

The measurement of spectral hemispherical emissivity can be performed using a BRDF (Bidirectional Reflectivity Distribution Function) apparatus [20] (similar to a goniometer module), scanning every incident and reflection angle of the hemisphere, often provided for a single wavelength defined by the laser source wavelength.

The calculation of total hemispherical emissivity is possible using an integrating sphere and a very wide band source and detector. Choosing the BRDF option [21] is still possible, but with a source and detector which wavelength is adjustable.

To sum up, indirect methods are rather simple to set up for the measurement of spectral directional emissivity but turn out to be optically complicated for hemispherical reflectivity measurement.

\subsubsection{Direct methods}

Direct radiometric method techniques are well suited for high temperature since they usually involve less optics [22]. They are based on the direct evaluation of the definition of the emissivity provided in equation 3. The main difficulty is to ensure that the sample and the blackbody exhibit the exact same 
temperature. Another difficulty is to maintain a homogeneous temperature across the sample measurement field.

For the spectral directional emissivity, the radiation is detected in a unique direction or a small solid angle by sensors and the sample temperature is controlled which thermocouples [23], pyrometer [24], IR camera [25], pyroreflectometer [26] or the use of Christiansen point [27]. Literature also provides several examples of heating techniques: thermostatic fluid [28], laser [24], electrical [23] or radiative heat sources [26]. Finally, the reference body can be either a laboratory blackbody [29] or a known high emissive sample [30].

An example of direct method is the so-called furnace drop technique proposed by Dozhdilkov et al. [31]. This technique consists in inserting the sample inside a commercial cavity blackbody until the sample temperature stabilizes. Then, the blackbody is dropped (meaning that the sample is rapidly extracted from the cavity) and the sample radiance is measured. Knowing the blackbody radiance at the same temperature, emissivity can then be deduced following Eq.3.

As mentioned in the title of Dozhdilkov et al. paper, this technique uses transient thermal states and is therefore not suited for high conducting materials. Another significant drawback is the inability to perform a continuous measure of emissivity and therefore investigate oxidation phenomenon.

A more recent development is the work of the LNE laboratory [26]. The sample is placed in a vacuum chamber and heated up by 7 IR halogen lamps through a quartz hemispherical dome. Signal emitted by the downside face of the sample is directed toward an IRTF spectrometer. Measurements can be performed for a bandwidth between 0.8 and $10 \mu \mathrm{m}$. The reference blackbody is a SiC cavity whose temperature is controlled by a $S$ thermocouple. A pyroreflectometer[32] is used to measure the sample temperature. Such radiative sources enable versatile heating with steep temperature rises. A maximum operating temperature of $1500^{\circ} \mathrm{C}$ is reported.

The latest development of direct methods facilities is detailed in a work of the Beijing Institute [33]. The back side of the sample is heated up by a furnace $\left(T<1000^{\circ} \mathrm{C}\right)$ while the front side faces a parabolic mirror. The mirror is setup on a rotary stage in order to face alternatively the sample and the cavity of a commercial blackbody. The emitted flux is then directed in an InGaAs detector which only allows an emissivity measurement over the NIR (0.8 to $2.2 \mu \mathrm{m}$ ) bandwidth.

It is worth noticing that the last two techniques do not allow an easy evolution toward measurement under inert gas atmosphere, they are therefore hardly applicable in the field of high temperature metals.

Another device has also been developed recently by CEMHTI [34]. An interesting particularity of this device is that it can be applied to semitransparent materials. A normal spectral emissivity can be measured for a wide bandwidth $(0.5$ to $500 \mu \mathrm{m})$ through two spectrometers. Sample is heated by a ceramic plate heater for $\mathrm{T}<1200 \mathrm{~K}$ and by a $\mathrm{CO} 2$ laser for higher temperatures which allows measurements between 700 and 2500K. Advantages of this device are the wide temperature range, wide bandwidth which allows calculating integrated emissivities and an application for both opaque and semitransparent materials. Sample temperature measurement is done through Christiansen point which is a wavelength where emissivity is equal to 1 for dielectric materials. For metallic samples, a thermocouple or the use of $\chi$ point (for pure metals) which is a wavelength where 
emissivity is invariant versus $\mathrm{T}$ can be used to determine sample temperature. Drawbacks are the complexity of the heating system with a set of mirrors to homogenize the sample temperature for the laser configuration. Another drawback is temperature measurement for metallic sample that don't have Christiansen point and implies the knowledge of the $\chi$ point.

Total directional emissivity can be obtained by numerical integration of spectral directional emissivity. Another issue would be to use a very wide bandwidth detector comparing the integrated flux of the sample with the one emitted by a blackbody at the same temperature.

For the measurement of spectral hemispherical emissivity, an integrating sphere is required to detect the emitted flux in the entire hemisphere. Another set-up would be a goniometer apparatus enabling the measurement of the emitted flux for different directions[35]. A spatial integration would then be necessary to retrieve the hemispherical quantity.

Finally, the total hemispherical emissivity can be obtained with an integrating sphere operating over a very wide spectral range or a goniometer module measurement the radiation for every directions and every wavelengths [36]. However, the most famous and certified method for evaluating this quantity is still the well-known calorimetric method [37],[38]. These methods are interesting because the total hemispherical emissivity can be directly measured. For this method the sample is heated to a fixed temperature (Ts) with an electrical resistance. The sample is then inserted into a black vacuum chamber with the internal wall temperature (Tw) maintained by liquid nitrogen or liquid helium. The power applied (P) to the sample is adjusted to maintain a constant temperature (Ts). The vacuum chamber enables to nullify the convective heat exchange. Emissivity can then be calculated by the following formula (eq. 11):

$$
\varepsilon=\frac{P}{S * \sigma *\left(T_{S}^{4}-T_{w}^{4}\right)}
$$

Where $\sigma$ is the Stefan-Boltzmann constant and $S$ stands for the sample surface area. The main advantages of the calorimetric method are the simplicity of the measurement principle and the fact that the total emissivity is easily deduced for several temperatures. Moreover, transient measurements can also be performed. The main drawback being that no spectral information can be obtained from such experiment. In addition, some experimental issues have led to reconsider such approaches. Indeed it is often difficult to maintain a homogeneous temperature over the sample, the stabilization time can be significant, and maintaining the environment (especially the walls temperature) can be very challenging.

\subsection{Method selection}

The choice of the method, based on the requirements of the measurement, is performed by comparison between several criteria like complexity of the system, atmosphere versatility... Some methods like integrating sphere were automatically eliminated due to the complexity of the adaptation to high temperature. 


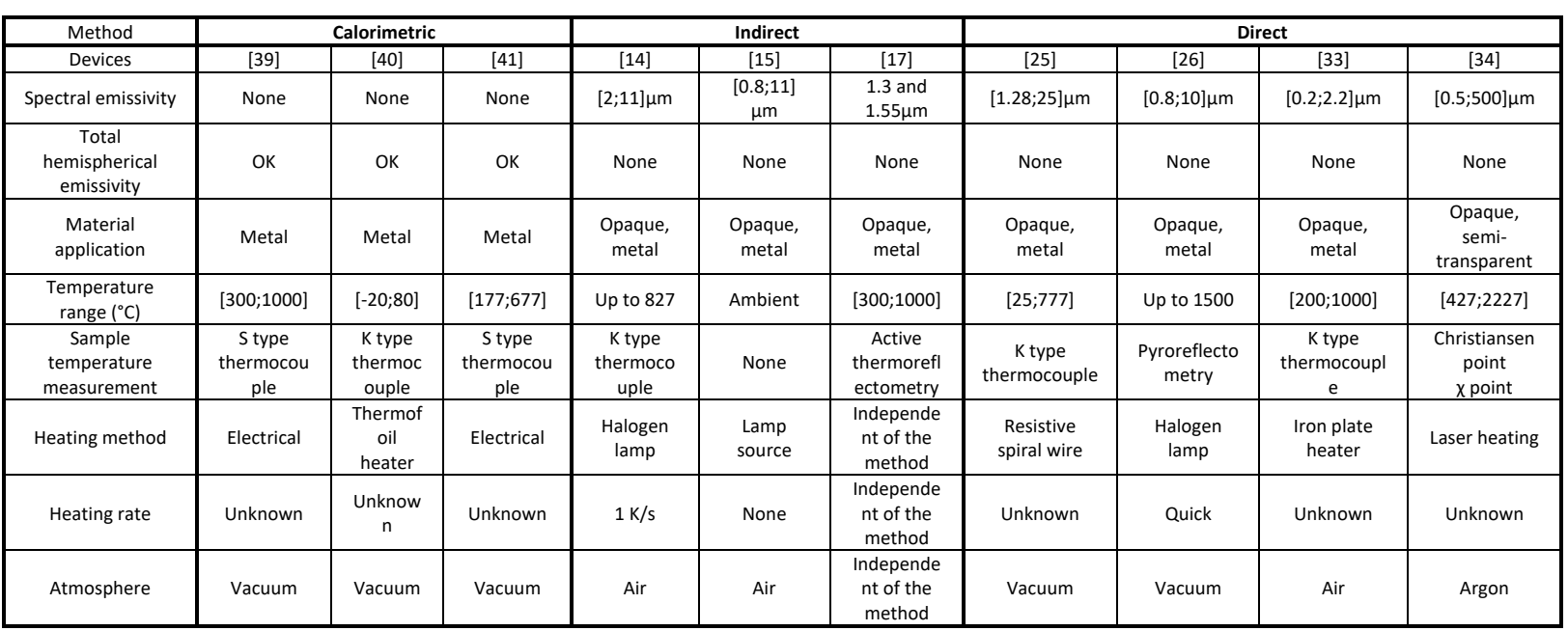

Table 2 : Comparison of emissivity measurement methods[39][40][41][14][15][17][25][26][33][34]

The aim of the proposed device here is to allow measurement of both spectral and integrated emissivity at high temperatures. Under such conditions, the calorimetric methods can be discarded since they are not suited for spectral applications. Direct methods devices are more adapted for high temperature applications and are usually simpler in their conception comparatively to indirect method devices. The choice is then made for a direct method based device with IR heating system to have high and flexible heating rate, even if devices are usually built-in and weighty. The difficulty then lies in the development of a standalone, fast, user-friendly, versatile and cheap device able to provide both spectral and integrated emissivity measurements. IR lamp is chosen (rather than laser) to simplify the system and to lower its cost. Moreover, IR heating is more suited to metallic materials than laser heating. Indeed, thanks to its wide emission spectrum, IR lamps focus more on the spectral domain where absorption is high (short wavelengths). Moreover, because of its high power spectral density, laser heating induces the risk to burn or modify the sample surface, which is penalizing for the measurement. In addition, such heating principle is very close to classical industrial heating systems (in term of chromaticity, heating rates, surface power...).

\section{Design and development of the device}

\subsection{Global view of the system}

An existing heating device used previously for several applications like for example pyrolysis [42] has been modified for direct method based emissivity measurement application. This device (Figure 1) consists of an elliptical high reflecting stainless steel surface. A radiative source is located at one focal point of the ellipse while the sample stands at the other focal point. The sample temperature is monitored by a thermocouple and can be controlled either manually or using PID controller adjusting the lamp input power. Sample heating method by an elliptical furnace allows high heating rate, ramp temperatures, cycling and temperature bearing and its design allows to easily switch between different atmospheric conditions. The external walls of the ellipse are maintained at ambient temperature by circulating water whose temperature can be controlled. Water can also be easily replaced by liquid nitrogen on the cooling system. 

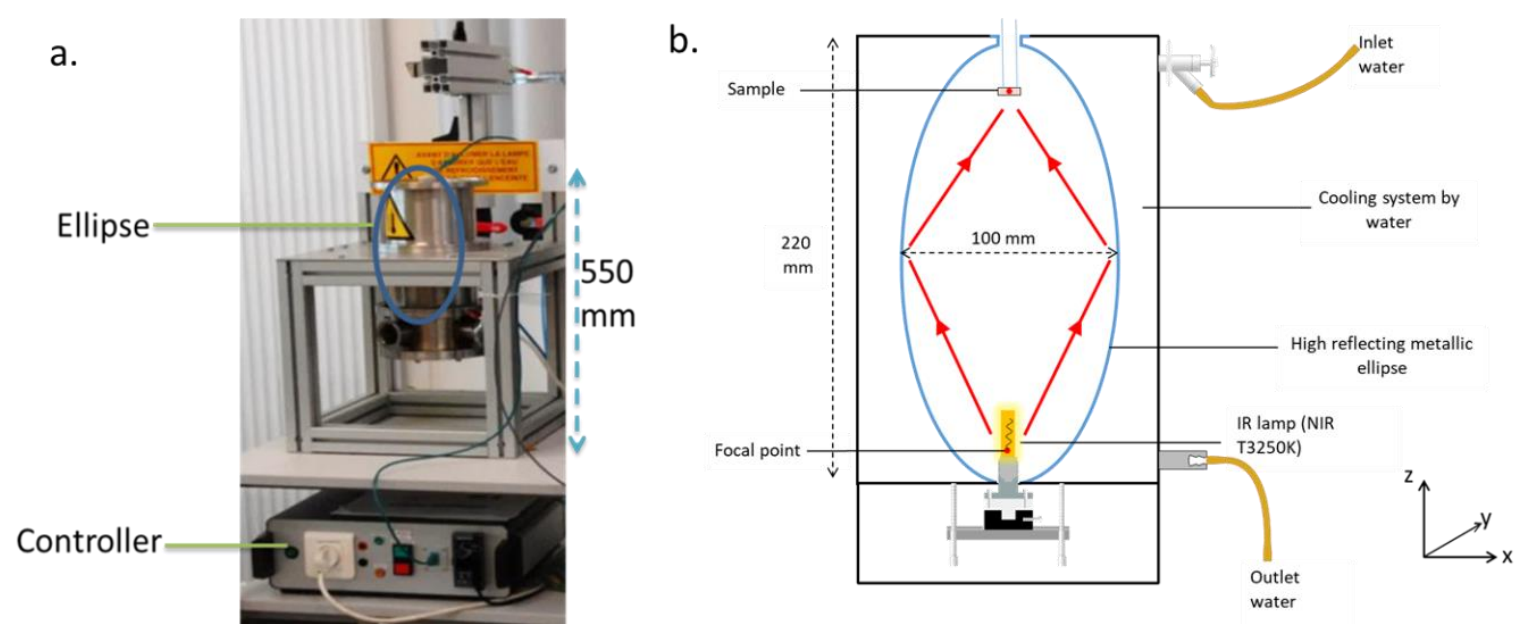

Figure 1 : Device original design: photograph (a), cross-section view (b)

In practice, the radiative source is significantly larger than a point and its accurate positioning at the focal point is challenging. Hence, a position adjustment system is added in order to allow translations of the lamp along $x, y$, and $z$ axis. A rotation around $x$ and $y$ is also made possible in order to account for possible filament disorientations. The emission of the heated sample is then measured on its top (back) face using either a NIR spectrometer equipped with a high temperature resisting fiber optics or with IR cameras (through a tilted flat Ag mirror). A turbomolecular vacuum pump is available for the device allowing measurements under secondary vacuum environment.

\subsection{Characteristics of the radiative source}

As mentioned above, the light source geometry needs to be known precisely in order to estimate its projection at the image focal point and therefore to assess the incoming heat flux on the sample. For most commercial high power lamps, the filament geometry consists in a vertical spiral. Considering the present application, the choice naturally comes down to an IR high power halogen lamp with a long vertical double-coiled filament. The IR lamp used in this work is manufactured by USHIO ${ }^{\circledR}$, has a nominal power of $1000 \mathrm{~W}$, a filament length of $25 \mathrm{~mm}$, a filament diameter of $4.5 \mathrm{~mm}$ and a nominal color temperature of 3200K. The maximum emission corresponding wavelength calculated is $906 \mathrm{~nm}$. The choice for a high power lamp allows having a wide range of heating rate on the sample. An infrared image (Figure 2) of the filament is captured with a NIR [0.9-1.7] $\mu \mathrm{m}$ camera XENICS ${ }^{\circledR}$ Xeva $^{2}$ equipped with a macro lens to evaluate the maximum emission area and to observe emission gradients along the filament length. 


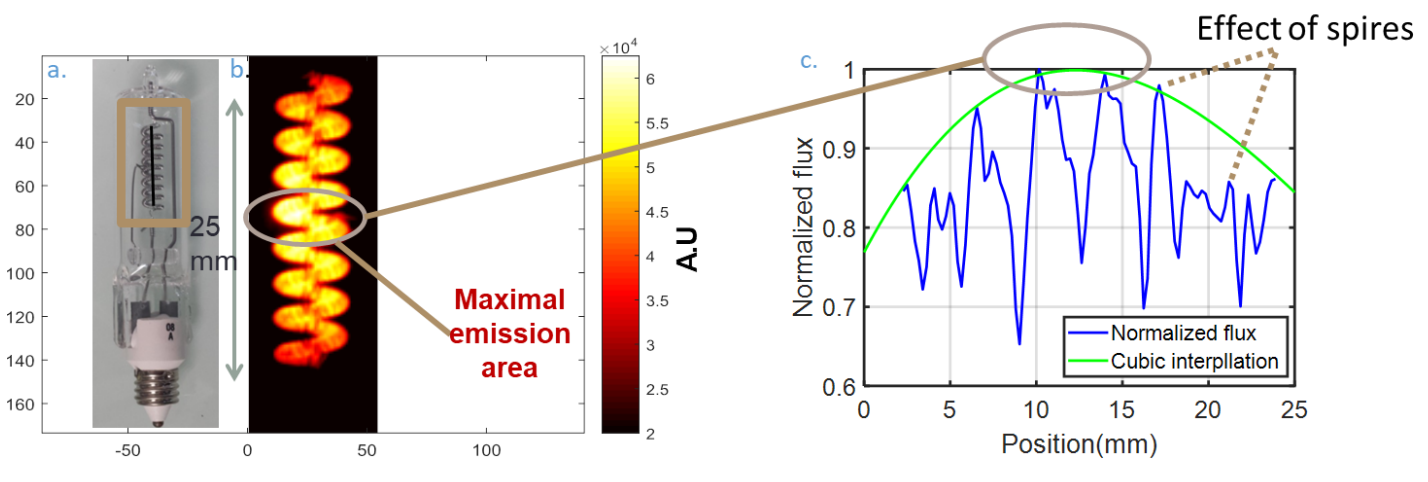

Figure 2 : Lamp filament emission: photograph (a), infrared image (b), Normalized flux (c)

It is seen in Figure 2 that the maximum emission area is located at the filament center and a maximal difference of $15 \%$ is observed between center and extremities of the filament. The difference is only of $5 \%$ in the four spires located at the center which corresponds at a length of $11 \mathrm{~mm}$. Such dimensions improve the positioning flexibility of the sample with respect to the source. Indeed the illuminated zone in the vicinity of the image focal point is larger.

\subsection{Design of the sample holder}

\subsubsection{Determination of sample dimensions}

The optimum diameter of the sample is computed from a Ray Tracing technique as in reference [11] [43]. Indeed, the incoming irradiance over the sample needs to be as high as possible (to reach the target temperature of $1200^{\circ} \mathrm{C}$ ), but above all, as homogeneous as possible. A first order approximation allows assessing the temperature of the sample from its response to a constant incoming flux on one side from:

$$
T(t)=T_{0}+\frac{2 P_{E}}{h_{g} \pi d^{2}}\left(1-e^{-\frac{t}{\tau}}\right)
$$

Where $T_{0}$ is the initial sample temperature, $d$ the sample diameter, $P_{E}$ the effective incoming flux on the sample, $h_{g}$ the global heat transfer coefficient, $d$ the sample diameter and $\tau$ the time constant. It therefore comes that the maximum temperature at steady state, is given by:

$$
T_{\text {max }}=\lim _{t \rightarrow+\infty} T(t)=T_{0}+\frac{2 P_{E}}{h_{g} \pi d^{2}}
$$

The global heat transfer coefficient $h_{g}$ is approximated for the sum of the convective and radiative exchange coefficients [44]. The Ray Tracing model is used to assess the effective incoming power $P_{E}$ from the following equation:

$$
P_{E}=P_{n o m} \cdot \alpha \cdot \beta
$$

Where $P_{\text {nom }}$ is the nominal (input) power of the lamps, $\alpha$ is the sample absorptivity and $\beta$ is the ratio of the incoming flux on the sample over the lamp's emitted flux and is defined as:

$$
\beta=\frac{\sum_{i=1}^{N_{t}} P_{s}(z) \cdot \rho_{w}{ }^{r i}}{\sum_{i=1}^{N_{t}} P_{s}(z)}
$$

where $N_{t}=10^{7}$ is the total number of computed rays, $\rho_{w}=0.97$ is the ellipse walls reflectance and $r i$ stand for the number of reflections of the $\mathrm{i}^{\text {th }}$ ray. Indeed, each ray leaves the lamp with a normalized power ranging from 0.75 to 1 according to the vertical positon $z$ of the starting point and 
the measured flux depicted in Figure 2. This power decreases after each reflection on the ellipse walls. In order to describe the possible heterogeneity of temperature over the sample, the power ratio $\beta$ can be discretized along the sample radius $r$ to compute the power ratio received at each location. For this purpose, the sample radius is divided into 50 cells and $\beta_{j}$ is assessed from

With

$$
\beta_{j}=\frac{\sum_{i \in N_{j}} P_{S}(z) \cdot \rho_{w}^{r i}}{\sum_{i \in N_{j}} P_{S}(z)}
$$

$$
\beta=\sum_{j=1}^{50} \beta_{j}
$$

Where $N_{j}$ is the set of rays that hits the $\mathrm{j}^{\text {th }}$ cell of the sample.

The lamp filament is modelled as a $25 \times 4.5 \mathrm{~mm}^{2}$ cylinder and the power assigned to each ray depends on its starting position along the $z$ axis. Four cases are treated as depicted in Figure 3 . The first case is the general case where the ray ends up on the sample after $r i$ reflections, the second corresponds to a number of reflection leading to decrease the ray power below $10 \%$ of its initial value (i.e. 76 reflections with $\rho_{\text {ellipse }}=0.97$ ), in the third and fourth cases, the ray hits either the sample holder or the bulb base. In cases 2 to $4, r i$ is set to $r i=+\infty$ i.e. the rays is discarded form the power computation.

Simple considerations lead to consider that the flux ratio $\beta$ depends on both the sample diameter and its vertical $(\mathrm{z})$ position with respect to the focal point (denoted $\delta$ ). Indeed, as depicted in Figure $3 e$, the vertical shift $\delta$ from the focus point leads to flatten the flux (defocusing effect) and modify the distribution of the $\beta_{j}$. Consequently, a total of 72 combinations of the sample positions ( $\delta=$ $[+5 ;-40])$ and diameters $(D=[10 ; 20])$ are tested. A minimal diameter of $10 \mathrm{~mm}$ is imposed by the necessity to weld a thermocouple on the sample back face and to get enough surface on the sample for optical measurement.

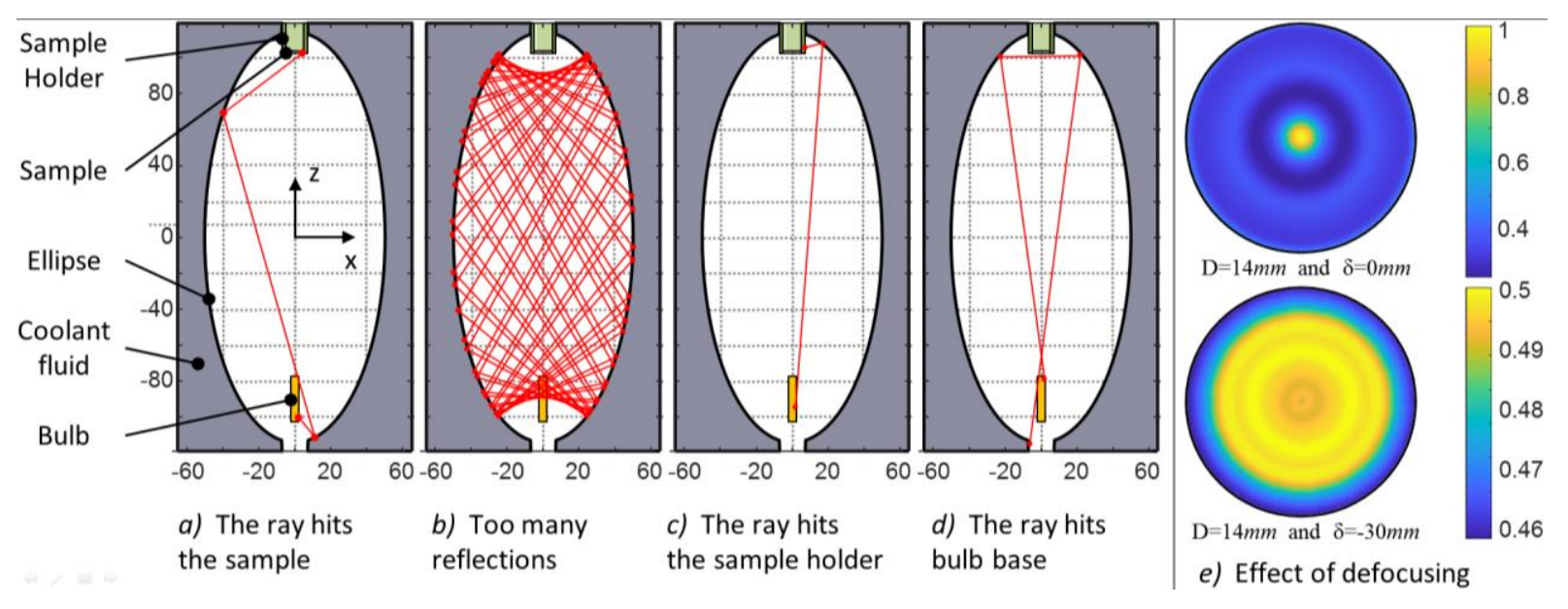

Figure 3: $(a-d)$, ray tracing for several cases. (e) radial distribution of $\beta_{j}$ : defocusing effect

Figure 4.a depicts the flux ratio $\beta$ for various diameters and vertical offsets, Figure 4.b it standard deviation along the radius of the sample (i.e. the flux homogeneity over the sample) and the Figure 
$4 \mathrm{c}$ depicted the computed steady state temperature assessed from equation (13). $\alpha$ value is set at 0.5 and a $h_{\mathrm{g}}$ value of $122.5 \mathrm{~W} \cdot \mathrm{m}^{-2} \cdot \mathrm{K}^{-1}$ is computed [44].
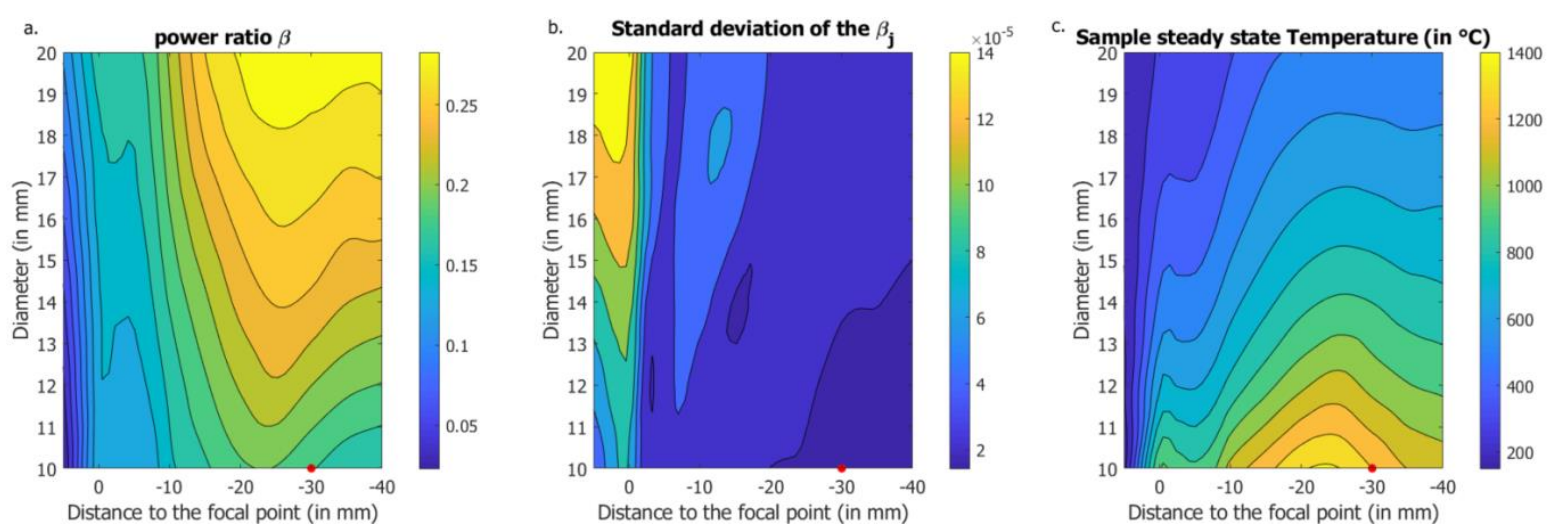

Figure 4: Flux ratio for several configurations (a), flux homogeneity over the sample (b), computed steady-state temperature (c)

It is seen from Figure 4 that increasing the sample diameter is not relevant. Indeed it leads to decrease the temperature with no gain on the spatial homogeneity of the incoming flux. In addition, defocusing of $10 \mathrm{~mm}$ to $30 \mathrm{~mm}$ significantly strengthen the homogenous flux hypothesis. Accordingly, in the following, a sample diameter of $D=10 \mathrm{~mm}$ and a distance to the focal point of $\delta=-30 \mathrm{~mm}$ are chosen.

\subsubsection{Sample holder characteristics}

A sample holder is required to position and maintain the sample within the cavity. The main expected characteristics are a good resistance to high temperatures $\left(1200^{\circ} \mathrm{C}\right)$, a low thermal conductivity (to decrease thermal gradients within the sample), a low thermal expansion coefficient and optical opacity (to avoid radiation lamp interference on the measured face of the sample). The material selected to abide by those conditions is a soapstone refractory ceramic. The sample holder is designed to center a $10 \mathrm{~mm}$ diameter sample equipped with a thermocouple. Ceramic wedges are used to set precisely the vertical position of the sample. As an example to validate heating performances, a platinum sample put on the optimal position defined in 2.3.1 part is heated with the IR lamp power set at $100 \%$. The sample reaches a maximal temperature of $1100^{\circ} \mathrm{C}$ at a rate of $8^{\circ} \mathrm{C} / \mathrm{s}$. In Figure 5, temperature gradient across the sample are depicted for a targeted temperature of $1000^{\circ} \mathrm{C}$ (measurement are made using a BII FLIR SC7000 MIR camera). The tolerable gap between the maximum and the minimum temperature of the measuring zone is set to $10^{\circ} \mathrm{C}$. Therefore, a $3.5 \mathrm{~mm}$ diameter circular maximal area is defined as the emissivity measurement zone. 

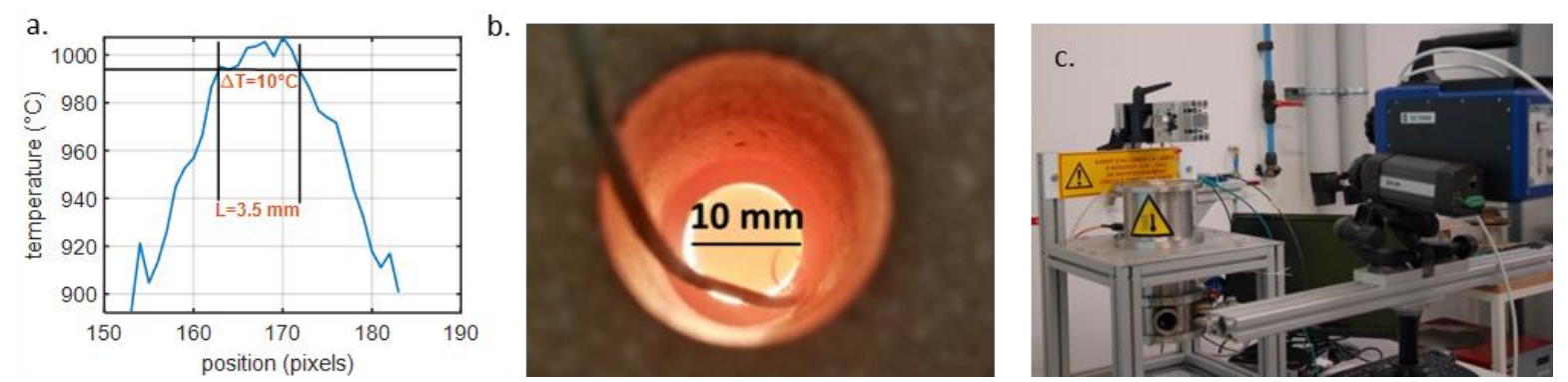

Figure 5 : Temperature gradient on the sample: experimental set-up (a), photograph of the heated sample (b), maximal area defined for emissivity measurement (c)

In this section, the requirements of the measurement have been achieved thanks to a numerical Ray Tracing approach, confirmed by experimental measurements. The diameter of the sample, the position to the focus point and the ability to reach a temperature of $1100^{\circ} \mathrm{C}$ have been demonstrated.

\begin{tabular}{ccccc} 
& Sample & & \multicolumn{2}{c}{ Temperature } \\
\hline Dimension & $\begin{array}{c}\text { Optimal } \\
\text { Position }\end{array}$ & $\begin{array}{c}\text { Sample } \\
\text { holder } \\
\text { material }\end{array}$ & $\begin{array}{c}\text { Maximal } \\
\text { value }\end{array}$ & $\begin{array}{c}\text { Heating } \\
\text { rate }\end{array}$ \\
\hline $\begin{array}{c}10 \mathrm{~mm} \\
\text { diameter }\end{array}$ & $\begin{array}{c}30 \mathrm{~mm} \text { from } \\
\text { focal point }\end{array}$ & Soapstone & $1100^{\circ} \mathrm{C}$ & $8^{\circ} \mathrm{C} / \mathrm{s}$ \\
\hline
\end{tabular}

Table 3 : Defined sample characteristics and temperature attainments

\section{Calibration and instrumentation of the system}

\subsection{Selection of the sensors for a wide band detection}

The measurement of spectral emissivity from direct method requires the most suited selection of sensors as implied by Wien's displacement law (eq.1). Moreover, as mentioned above, the hot forming applications targeted in the present paper lead to focus on temperature range [600-1000] ${ }^{\circ} \mathrm{C}$. The blackbody radiation calculated by Planck's law and their corresponding derived curves for these temperatures are shown in Figure 6. 

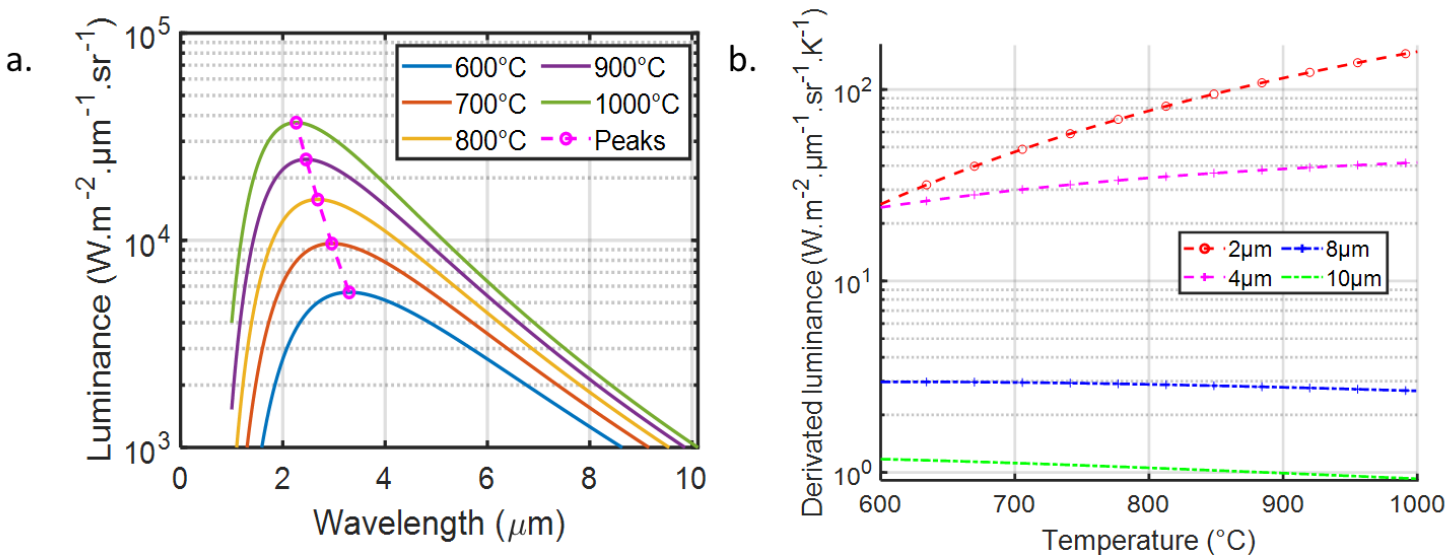

Figure 6 : Blackbody emission (a) and derived curves (b)

For temperatures of $600^{\circ} \mathrm{C}$ and $1000^{\circ} \mathrm{C}$, wavelengths corresponding to maximum emission are respectively found at 2.26 and $3.31 \mu \mathrm{m}$ which implies emitted radiations respectively between [1.1311.3] $\mu \mathrm{m}$ and [1.65-16.5] $\mu \mathrm{m}$. Therefore, investigating integrated emissivity requires the whole classical ranges of IR sensors: namely band I for NIR, and bands II and III for MIR. The derived curves depicted in Figure 6.b) show the high sensitivity of NIR BI wavelengths at high temperature and conversely the low sensitivity for MIR BIII. An optimal emissivity measurement would be done through a continuous sensor operating between 1.1 and $16.5 \mu \mathrm{m}$. However, the device requirement for standalone measurements and the sensors availability dictate a discontinuous measurement between 1.3 and $10 \mu \mathrm{m}$. The design device still allows to easily change sensors with thermopile, photodiode...

For NIR measurements, a FT-IR spectrometer NeoSpectra ${ }^{\circledR}$ operating at $[1.3 ; 2.5] \mu \mathrm{m}$ equipped with a single PbS photodetector is used. The spectrometer input plug is connected to a high temperature resistant optical fiber that is put very close to the sample (less than $3 \mathrm{~mm}$ ). This fiber can operate between -20 and $1000^{\circ} \mathrm{C}$. For MIR BII measurements, an IR camera FLIR SC7000 equipped with a filter wheel is used. This camera has a resolution of $320 * 256$ pixels with an image frequency from 1 to 500 $\mathrm{Hz}$. Integration time can be adjusted between $1 \mu \mathrm{s}$ to around 1s. Its spectral range spreads from $2.5 \mu \mathrm{m}$ to $5.5 \mu \mathrm{m}$. The sensor is also cooled by an internal stirling system. For MIR BIII measurements, an IR camera FLIR SC325 is used. This camera has a resolution of $320 * 240$ pixels with an image frequency of $30 \mathrm{~Hz}$ and a spectral range between 7.5 and $13 \mu \mathrm{m}$. BII and BIII cameras will be equipped with filters described in Table 4.

\begin{tabular}{|c|c|c|c|c|}
\hline Camera & \multicolumn{3}{|c|}{ MIR BII } & MIR BIII \\
\hline Wavelength $(\mu \mathrm{m})$ & 3,027 & 4 & 5,071 & 10 \\
\hline Bandwidth $(\mathrm{nm})$ & 60 & 80 & 96 & 2000 \\
\hline $\begin{array}{c}\text { Transmission at } \\
\text { peak (\%) }\end{array}$ & 73.91 & 60 & 68.86 & 92 \\
\hline
\end{tabular}

Table 4 : Optical filters characteristics

MIR BII filters wavelengths are selected to have a regular distribution on the SC7000 camera bandwidth with three bandpass filters allowing measurements respectively at the beginning, the center and the end of the camera bandwidth. For MIR BIII measurements, a sole bandpass filter with 
a wavelength corresponding at SC325 camera bandwidth center is selected. The discontinuity on the measurement caused by this filter selection will then be studied in part 3.2.

\subsection{Influence of spectrum discontinuity on integrated emissivity}

The goal of the presented experiment is to provide both spectral emissivity and integrated emissivity. In practice, the integrated emissivity is calculated from the spectral emissivity measurement. However, such computation usually relies on the hypothesis of radiance continuity over the emission spectrum which can hardly be satisfied with the sensors selection presented in section 3.1.

Accordingly, the discontinuities of the obtained spectrum lead to an integration error on the integrated emissivity value. It is here proposed to assess such error from the comparison of integrated emissivity calculated from continuous or discontinuous spectrums. The chosen reference material is polished Platinum for which the spectral normal emissivity can be calculated from its optical indices [45] and the radiative properties slightly depends on temperature. Figure 7 shows the obtained continuous spectrum, the detection bands of the NIR spectrometer and the camera filters bands are also highlighted. The missing points in-between these bands are interpolated using either linear, cubic or Akima splines interpolant[46]. The corresponding emissivity spectrums are then integrated using (6) and compared to the continuous integration (see Table 5), with the Platinum spectral emissivity chosen at room temperature.

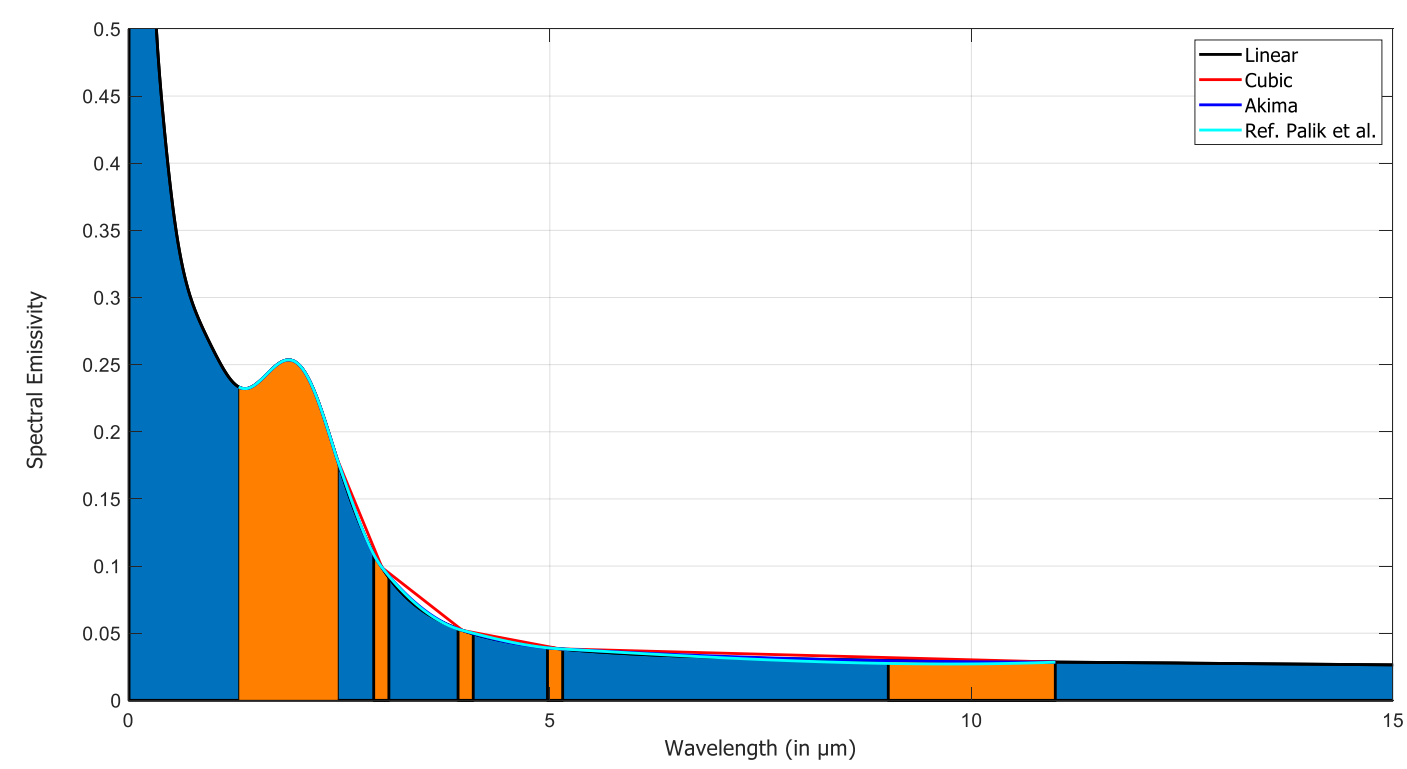

Figure 7: Theoretical spectral emissivity for polished platinum [45] and interpolated values with linear, cubic and Akima spline interpolant

In is seen from Table 5 that the integrated emissivity calculated from the Akima spline interpolant exhibits lower errors than other approaches and remains always below $0.2 \%$. Accordingly this latter interpolation will be used in the present study.

Another significant source of error is the bounds of the integration. Indeed the chosen sensor impose the integration over $1.3 \mu \mathrm{m}$ to $11 \mu \mathrm{m}$ while the bound of Eq. 6 are 0 and +inf. Using the data proposed by Palik et al. [45], the discrepancy between the two integrated values of emissivity can be assessed to $4.7 \%$ and $0.37 \%$ for $600^{\circ} \mathrm{C}$ and $1000^{\circ} \mathrm{C}$ respectively. At the lowest temperature, the error mainly comes from the far infrared cut-off (at $10 \mu \mathrm{m}$ with the proposed setup). Such drawback can be tackle 
down be adding another sensor above $10 \mu \mathrm{m}$. For instance, the error comes down to $1.8 \%$ at $600^{\circ} \mathrm{C}$ with an extra point at $14 \mu \mathrm{m}$.

\begin{tabular}{|c|c|c|c|c|c|}
\hline \multicolumn{2}{|c|}{ Spectrum } & Palik [45] & $\begin{array}{c}\text { Linear } \\
\text { interpolant }\end{array}$ & $\begin{array}{c}\text { Cubic } \\
\text { interpolant }\end{array}$ & $\begin{array}{c}\text { Akima spline } \\
\text { interpolant }\end{array}$ \\
\hline \multirow{2}{*}{$\mathrm{T}=600^{\circ} \mathrm{C}$} & $\varepsilon$ & 0.0746 & 0,0773 & 0,0750 & 0.0747 \\
\cline { 2 - 6 } & $\Delta \varepsilon / \varepsilon(\%)$ & - & 3,8 & 0,59 & 0.10 \\
\hline \multirow{2}{*}{$\mathrm{T}=1000^{\circ} \mathrm{C}$} & $\varepsilon$ & 0.1185 & 0.1211 & 0.1192 & 0.1187 \\
\cline { 2 - 6 } & $\Delta \varepsilon / \varepsilon(\%)$ & - & 2.1 & 0.33 & 0.17 \\
\hline
\end{tabular}

Table 5 : Integrated emissivity calculation with continuous and discontinuous spectrums

To sum up, in this section, the choice of an optimized interpolation method (Akima spline interpolant) has been performed to compensate the use of spectral detectors and not total radiation ones. However, at low temperatures, the main source of error is the maximum detection wavelength of the system $(11 \mu \mathrm{m})$, which is below the maximum emission wavelength of thermal emission.

\subsection{Thermal calibration of sensors}

\subsubsection{Thermal calibration}

○ Thermal calibration of the NIR spectrometer

The spectrometer (equipped with an optical fiber (see section 2.)) is calibrated between 600 and $1000^{\circ} \mathrm{C}$ using a R1500T Land blackbody. Corresponding spectrums are represented in Figure 8.

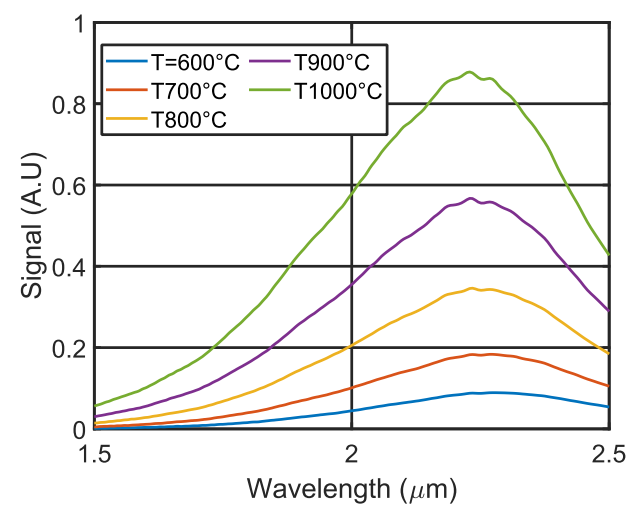

Figure 8 : NIR spectrometer calibration with primary blackbody

Spectrum values increase with temperature and a peak is observed at $2.2 \mu \mathrm{m}$ corresponding to the maximum spectral response of the photodetector. Emitted blackbody signal is multiplied by the photodetector spectral response and the fiber transmission which explained the curves' shape.

- Thermal calibration of the MIR camera SC7000

MIR camera equipped with three filters (around 3, 4 and $5 \mu \mathrm{m}$ ) is calibrated for each configuration (Figure 9a.). To avoid saturation, a signal value $\left(I_{d}\right)$ of 10000 digital levels is maintained by adjusting integrating time $\left(t_{i}\right)$. Ratio between digital levels under exposition time increases with temperature. For a monochromatic case, this ratio is supposed to decrease with wavelength unlike what is shown in Figure 9a. This shift can be explained by the difference of bandwidth and maximum transmission at peak between each filter (Cf. Table 4). 
Unlike SC7000 camera, exposition time is not adjustable on BIII camera SC325. Digital levels (b.) with a $10 \mu \mathrm{m}$ filter equipped on camera present a linear trend which implies a quasi-constant sensitivity with temperature over this range (see section 3.1). This trend seems in opposition with Planck's law, but this is only a scale effect. Enlarging the temperature range would lead to the classical exponential rise as seen in Figure 9 a.
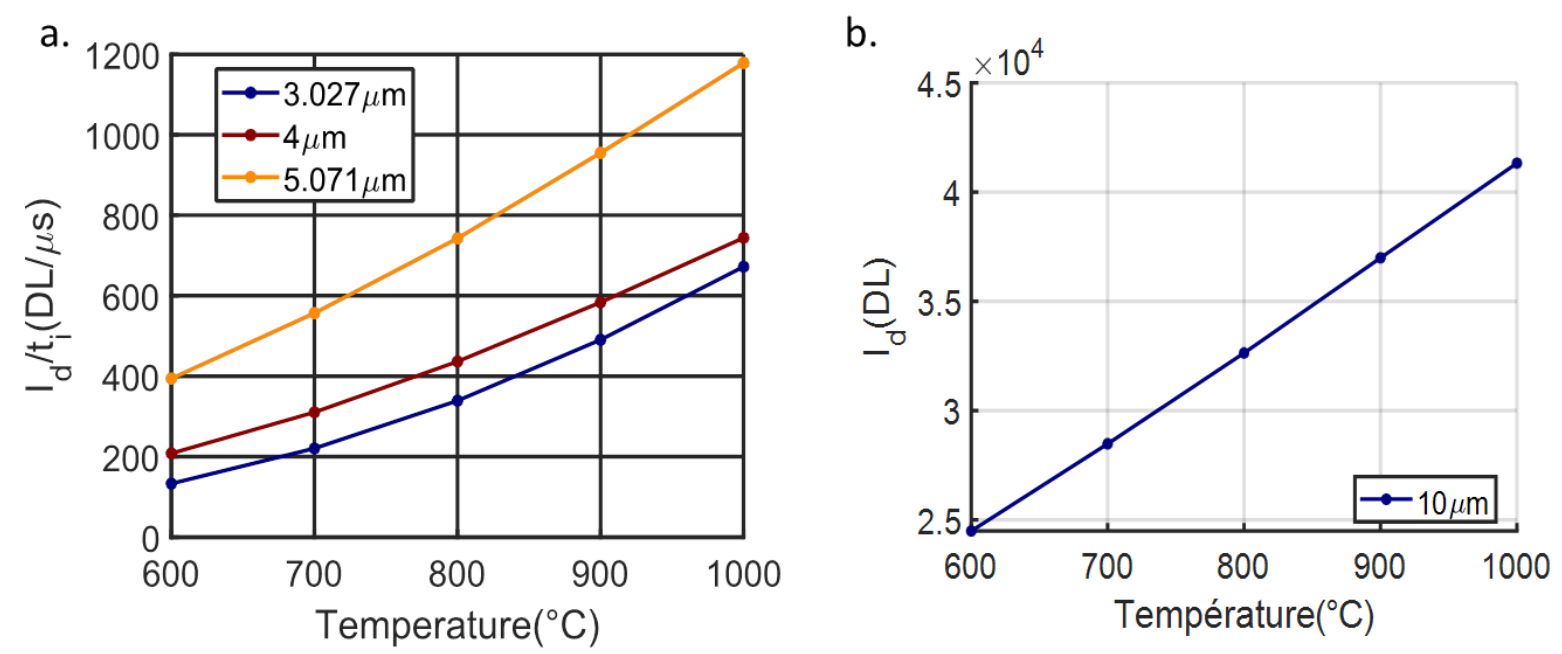

Figure 9 : a. MIR BII SC7000 camera calibration with blackbody, b. MIR BIII SC325 camera calibration with blackbody

\subsubsection{Measurement uncertainties}

Among the influence quantities governing the emissivity uncertainty (sensor noise, spurious signal, temperature uncertainty, sample thermal heterogeneity...), thermal effects are dominating. In this section, the uncertainty arising from a non-uniform temperature distribution over the sample and on the temperature measured value due to sensor uncertainty are investigated.

- Effect of non-uniform temperature distribution

Preliminary, this problem does not concern the MIR BII and BIII measurements, as they are performed using infrared cameras. Indeed, the thermal homogeneity of the surface over the projection of one pixel on the sample (around $500 \mu \mathrm{m}$ ) can be considered as satisfactory (see Fig. 2). On the contrary, the BI measurement performed by the means of an optical fiber with a nonzero acceptance cone must be discussed. The measurement is here performed on a small surface, with a possible thermal gradient on it, and the sensor integrates spatially the signal over such elementary surface

The optical fiber has an acceptance angle of $30^{\circ}$ and is put very close to the sample surface $(2.5 \mathrm{~mm})$, which implies a $1.38 \mathrm{~mm}$ diameter area for the measurement. According to Figure 5, the corresponding temperature gap is $\Delta \mathrm{T}=3^{\circ} \mathrm{C}$ (i.e. $\sigma_{\mathrm{T}}=1^{\circ} \mathrm{C}$ for a Gaussian distribution). Uncertainty on emissivity measurement arising from non-uniformity temperature can be evaluated through the following formula[47] : 


$$
\frac{\Delta \varepsilon}{\varepsilon}(N U)=\left|\frac{c_{2} /(\lambda T)}{\exp \left(-\frac{c_{2}}{\lambda T}\right)-1}\right| \frac{\Delta T}{T}
$$

This equation can also be written with standard deviations $\left(\Delta \varepsilon=3 * \sigma_{\varepsilon}\right.$ and $\left.\Delta \mathrm{T}=3{ }^{*} \sigma_{\mathrm{T}}\right)$ for a Gaussian distribution. This relative standard deviation is presented between 1 and $16.5 \mu \mathrm{m}$ in Figure 10.

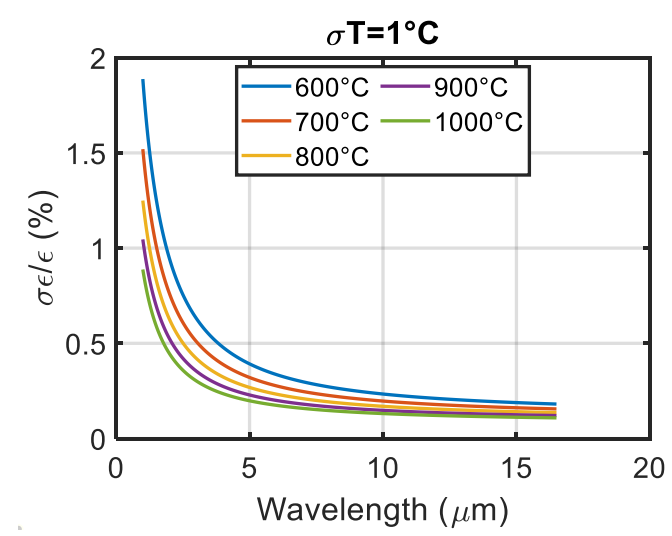

Figure 10 : Relative standard deviation on emissivity measurement arising from non-uniformity temperature between 1 and $16.5 \mu \mathrm{m}$ for a $3^{\circ} \mathrm{C}$ temperature gap

Non uniformity temperature has a higher impact on emissivity measurement in NIR range and lower in MIR range. It is also shown that the uncertainty on emissivity decreases slightly with temperature. The highest error is then recorded on the edges of the measurement area and is maximum at $600^{\circ} \mathrm{C}$, but remains inferior to $1.3 \%$ on the NIR spectrometer range and lower than $0.3 \%$ on the MIR range.

- Effect of temperature uncertainty (TU) on emissivity

In this work, the emissivity is calculated by a direct method, and is defined as the ratio of the detector signals $S(T)$ to the black body signal $\left(S_{0}(T)\right.$ set at the same temperature $\left.T\right)$. Hence, a bias on the temperature impacts the signals detected, so the emissivity evaluation, as depicted by the following formula:

$$
\frac{\sigma_{\varepsilon}}{\varepsilon}(T U)=2 * \frac{\sigma_{S}}{S}
$$

In order to link the signals and the temperature uncertainties, it is necessary to introduce the sensitivity of the detector, denoted $\mathrm{k}(\mathrm{T})$. This study is performed at wavelength $2.2 \mu \mathrm{m}$ for NIR spectrometer and $5.071 \mu \mathrm{m}$ for MIR BII camera, where sensitivity is maximum for each band, in order to maximize the uncertainty. . Moreover, as the NIR BI and MIR BII detectors response are non-linear, the sensitivity depends on the temperature, and the detectors response is approximated by a second-order polynomial (as the analytical formula is unknown).On the other hand, MIR BIII detector's response is approximated by a linear approximation. Temperature sensitivity is then deduced from raw signal spectrums.

Signal uncertainty can then be deduced from sensitivity and sensor uncertainty following this formula (for small variations of the temperature):

$$
\sigma_{\mathrm{S}}(\mathrm{T})=\mathrm{k}(\mathrm{T}) * \sigma_{\mathrm{T}}
$$

Where $\mathrm{k}$ is the temperature sensitivity $\left(\mathrm{A} . \mathrm{U} .{ }^{\circ} \mathrm{C}^{-1}\right), \sigma_{S}$ the signal standard deviation (A.U). 
The absolute error on temperature $\Delta \mathrm{T}$ is fixed by the chosen sensor (type $\mathrm{K}$ thermocouple). For such device, the manufacturer standard claims that the deviation evolves linearly in between 333 and $1200^{\circ} \mathrm{C}$ :

$$
\sigma_{\mathrm{T}}=0.0025 * \mathrm{~T}
$$

To sum up, the relative uncertainty on emissivity is given by the following formula:

$$
\frac{\sigma_{\varepsilon}}{\varepsilon}(T U)=2 * \frac{0.0025 * \mathrm{k}(\mathrm{T}) * \mathrm{~T}}{S(T)}
$$

To finish, the total uncertainties are obtained by the propagation of the standard deviations deduced from each cause of error, applying a coverage factor of 2.

$$
\frac{\sigma_{\varepsilon}}{\varepsilon}(T U)=2 * \frac{0.0025 * \mathrm{k}(\mathrm{T}) * \mathrm{~T}}{S(T)}
$$

Table 6 presents the maximum total uncertainty for each spectral band.

\begin{tabular}{|c|c|c|c|}
\hline Detector's range & NIR BI & MIR BII & MIR BIII \\
\hline Wavelength $(\mu \mathrm{m})$ & 2.2 & 5.071 & 10 \\
$\begin{array}{c}\text { Approximated detector's } \\
\text { response (A.U) }\end{array}$ & $2 * 10^{-6} * T^{2}-0.0022 * T+0.63$ & $0.0011 * T^{2}-0.27 * T-149.2$ & $42 * T-960$ \\
Sensitivity (A.U. $\left.{ }^{\circ} \mathrm{C}^{-1}\right)$ & $k(T)=4 * 10^{-6} * T-0.0022$ & $k(T)=0.0022 * T-0.27$ & $k(T)=42$ \\
Maximal uncertainty (\%) & $7.4\left(\right.$ at $\left.700^{\circ} \mathrm{C}\right)$ & $1.3\left(\right.$ at $\left.600^{\circ} \mathrm{C}\right)$ & $1.5\left(\right.$ at $\left.600^{\circ} \mathrm{C}\right)$ \\
\hline
\end{tabular}

Table 6 : Relative emissivity uncertainties arising from temperature uncertainty

Uncertainties due to non-uniform temperature distribution and temperature uncertainty are only relevant in NIR BI measurements. Indeed, uncertainties due to temperature do not exceed $1.5 \%$ in MIR BII and MIR BIII. Maximum combined uncertainties due to temperature effects in NIR band are observed at $700^{\circ} \mathrm{C}$ with a value of $7.4 \%$. However, this maximal uncertainty slightly decreases with temperature with a value of $5.4 \%$ at $1000^{\circ} \mathrm{C}$.

\subsubsection{Sample-holder contribution on the measurement}

As depicted in Figure 11 the chosen setup imposes to have the sample positioned into a sampleholder that absorbs a part of the flux emitted by the lamp and is also heated by the sample through thermal conduction. Accordingly, the sample holder own emission interferes with the measurement. Such emission can be spitted in three contributions as follows:

$$
\tilde{S}(T)=S(T)+S_{s h 1}\left(T_{s h}, \Omega_{1}\right)+\rho(T) S_{s h 2}\left(T_{s h}, \Omega_{2}\right)+S_{l}+S_{d}
$$

Where $S_{s h 1}$ is the emitted signal toward the sensor $\left(\Omega_{1}\right.$ being the viewing solid angle of the sample holder from the sensor), $S_{s h 2}$ is the signal emitted from the sample-holder and reflected upon the sample surface ( $\left(\Omega_{2}\right.$ is then the viewing solid angle of the sample from the sensor) and $S_{\text {sh } 3}$ is the complementary part of the emitted flux from the sample holder so that $\Omega=\Omega_{1}+\Omega_{2}+\Omega_{3}=2 \pi \mathrm{sr}$. 
Consequently, the signal received by sensors $\tilde{S}(T)$ (i.e. the measurement) is a sum of various contribution among which the emitted signal from the sample $S(T)$, spurious signal emitted from the sample-holder $S_{s h 1}\left(T_{s h}\right)$, the reflection of this latter emission over the sample surface $\rho_{\lambda}(T) *$ $S_{s h 2}\left(T_{s h}\right)$ (where $\rho$ stands for the sample reflectance), the signal leaked by the lamp through sampleholder gaps $S_{l}$ and the intrinsic sensor signal $S_{d}$ (detector offset). All those spurious signals, (summarized in Eq. 23) must be assessed to access the intended measure $\varepsilon(T)$ from $\tilde{S}(T)$.

One should notice that this equation reads differently for the two setups discussed in the above: the NIR configuration where an optical fiber is used and the MWIR configuration where an Ag mirror is used.

a.

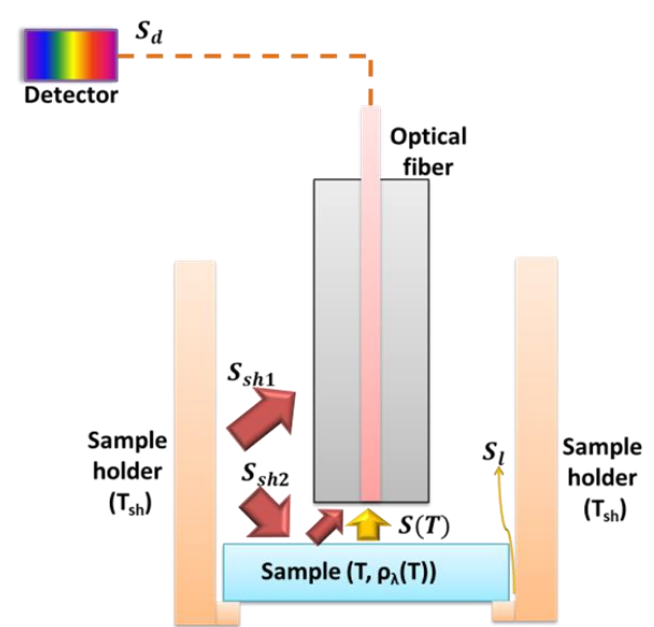

b.

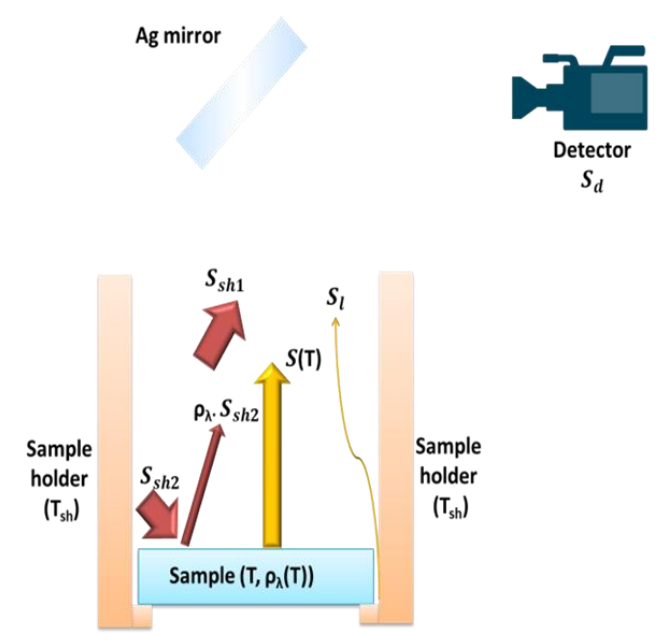

Figure 11 : Spurious signals illustration for: NIR configuration (a), MIR configuration (b)

When placed in front of a cavity blackbody set at a temperature $T$, the contribution of the sample holder is obviously null along with the spurious direct signal from the lamp $S_{l}$. The measurement reads:

$$
\tilde{S}^{0}(T)=S^{0}(T)+S_{d}
$$

Which allows the assessment of $S^{0}(T)$. Hence, equation (23) can be rewritten by replacing sample signal $S(T)$ by the product of spectral emissivity and blackbody emission $S^{0}(T)$ using Equation 2.

$$
\tilde{S}(T)=\varepsilon(T) S^{0}(T)+S_{s h 1}\left(T_{s h}\right)+\rho(T) S_{s h 2}\left(T_{s h}\right)+S_{l}+S_{d}
$$

Kirchhoff's law, applied to opaque materials, enables to link the emissivity to reflectivity as $\varepsilon=1-\rho$. Eq. 25 becomes:

And

$$
\tilde{S}(T)=\varepsilon(T) S^{0}(T)+S_{s h 1}\left(T_{s h}\right)+(1-\varepsilon(T)) S_{s h 2}\left(T_{s h}\right)+S_{l}+S_{d}
$$

$$
\varepsilon(T)=\frac{\tilde{S}(T)-S_{s h 1}\left(T_{s h}\right)-S_{s h 2}\left(T_{s h}\right)-S_{l}-S_{d}}{S^{0}(T)-S_{s h 2}\left(T_{s h}\right)}
$$


The variables $S_{s h 1}\left(T_{s h}\right), S_{s h 2}\left(T_{s h}\right), S_{l}$ and $S_{d}$ can be phrased as the contributions of all unintended emissions from the environment. Their evaluations depend on the selected setup (NIR or MWIR). $S_{s h 1}\left(T_{s h}\right)$, and $S_{s h 2}\left(T_{s h}\right)$ contributions on the measurement can be either computed by numerical simulation or evaluated experimentally. Nevertheless, boundary limits (sample holder temperature, sample temperature distribution, radiative properties) need to be known precisely for numerical computation. An experimental evaluation seems more appropriate and simpler for this set-up.

\section{- Environment contribution in NIR BI}

The use of the NIR spectrometer involves an optical path through an optical fiber. It has an acceptance angle of $30^{\circ}$ and is put very close $(2.5 \mathrm{~mm})$ to the sample surface. The projected measure therefore covers a $1.44 \mathrm{~mm}$ radius circle, hence fully included upon the sample surface. Accordingly, spurious signals originating from both the lamp $\left(S_{l}\right)$ and the direct emission of the sample-holder ( $\left.S_{s h 1}\left(T_{s h}\right)\right)$ can be neglected and discarded. It therefore comes:

$$
\varepsilon(T)=\frac{\tilde{S}(T)-S_{\text {sh2 } 2}(T)-S_{d}}{S^{0}(T)-S_{\text {sh } 2}(T)}=\frac{\tilde{S}(T)-S_{\text {sh2 }}(T)-S_{d}}{\widetilde{S^{0}}(T)-S_{\text {sh } 2}(T)-S_{d}}
$$

$S_{d}$ can be easily assessed from shielding the optical fiber from any incoming flux within its operating wavelength range. On the other hand, assessing $S_{s h 2}(T)$ requires a specific setup. Rewriting Eq.26 it comes:

$$
S_{s h 2}(T)=\frac{\left(\tilde{S}(T)-S_{d}\right)-\varepsilon(T) *\left(\widetilde{S^{0}}(T)-S_{d}\right)}{1-\varepsilon(T)}
$$

Therefore, a reference material whose emissivity is known precisely can be used to assess this sample holder signal contribution. This material has to be highly reflective to minimize direct signal emitted by the sample and ideally must have low emissivity variations with temperature. Polished platinum fills those conditions considering its chemical stability and low oxidation with temperature. Furthermore, the low dependence of emissivity platinum with temperature in NIR band has been shown (1.8 to $3.1 \mu \mathrm{m}$ for this work) by Deemyad and Silvera [48]. This statement is also confirmed by CEMHTI [34] in their works on platinum $\chi$ point. Under the hypothesis of a constant emissivity with temperature $\left(\varepsilon(T) \approx \varepsilon\left(20^{\circ} \mathrm{C}\right)\right)$, the emissivity of a polished platinum sample is measured at room temperature on a commercial IRTF spectrometer Bruker vertex 70 with the help of an infragold integrating sphere. This value is then used in Eq.28 to assess $S_{s h 2}(T)$. Figure 12a depicts the evolution of such contribution with respect to temperatures $T$ ranging from $600^{\circ} \mathrm{C}$ to $1000^{\circ} \mathrm{C}$. 
a.

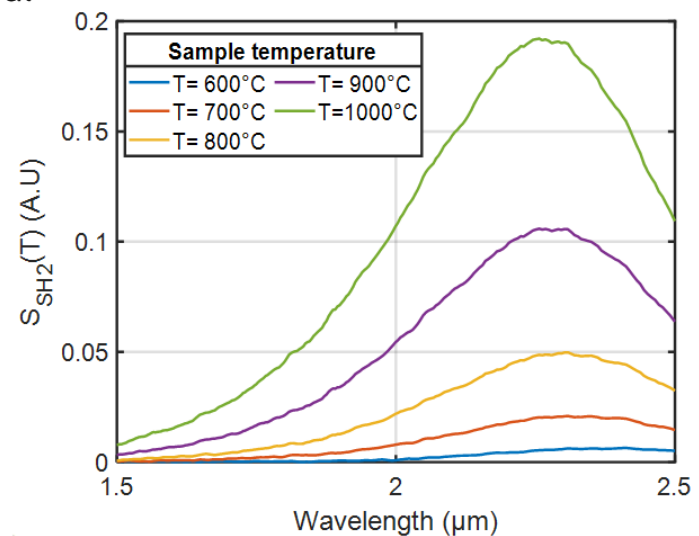

b.

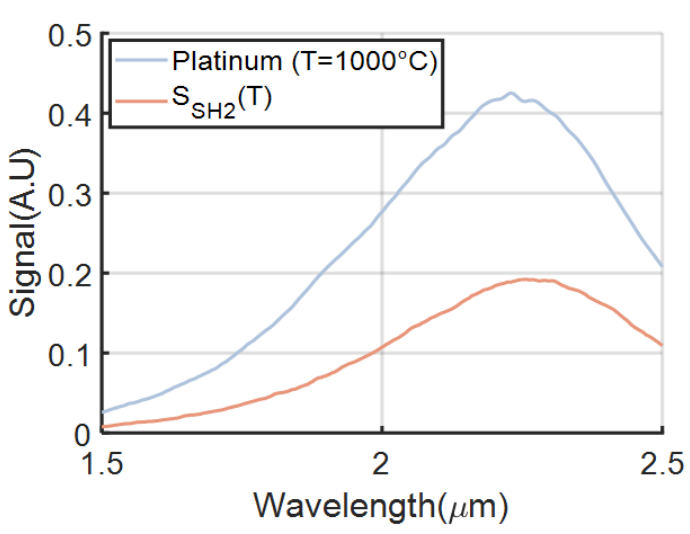

Figure 12 : Spurious sample holder signal spectrums (a) and comparison with platinum sample emission at $1000^{\circ} \mathrm{C}(\mathrm{b})$

Sample-holder emission increases with temperature and corresponding signal curves have the same shape than the sample's one. The Figure $12 \mathrm{~b}$ also shows that this spurious contribution cannot be overlooked especially at high temperature with a value close to $50 \%$ of the signal emitted by the sample at $1000^{\circ} \mathrm{C}$.

- Environment contribution in MIR BII and BIII

In this configuration, the cavity of the sample-holder is empty allowing any spurious signal to reach the sensor. It is chosen to use a cooled metallic pipe to discard the contributions of the sample holder and the lamp $S_{s h 1}=S_{l}=S_{s h 2}=0$. In practice, the pipe needs to be cooled in order to prevent any emission from it. Therefore, once the targeted temperature of the sample is reached, the pipe is inserted and for a short period of time (around 3 seconds), the measured signal becomes:

$$
\tilde{S}(T)=\varepsilon(T) S^{0}(T)+S_{d} \Rightarrow \varepsilon(T)=\frac{\tilde{S}-S_{d}}{S^{0}}=\frac{\tilde{S}-S_{d}}{\widetilde{S^{0}}-S_{d}}
$$

Such approach implies a fast insertion of the pipe (around 1 second) and an instant measure afterward to avoid a new spurious signal arising from the pipe as it heats up.

\section{Results}

\subsection{Sample characteristics and experiment set up \\ - Sample characteristics}

Two platinum samples are used in the present paper. Sample $A$ is used for environment influence computation and Sample B is used for device validation. Both exhibit a commercial purity of $99.95 \%$. The sample $A$ is polished with a $2 \mu \mathrm{m}$ felt in order to increase its reflectance and then improve the environment influence calculation (cf. Eq.28). The sample B is processed as-received. Roughness measurements were made on both samples and are presented in Table 7. The value of Sa and Sq are presented along with the classical roughness $\mathrm{Ra}$ and $\mathrm{Rq}$ since they provide valuable information on the surface flatness (absent from the Ra and Rq calculations). 


\begin{tabular}{lccccccc} 
& \multicolumn{3}{c}{ Dimensions } & \multicolumn{2}{c}{$\begin{array}{c}\text { Waviness \& } \\
\text { Roughness }\end{array}$} & \multicolumn{2}{c}{ Roughness } \\
\cline { 2 - 8 } & $\begin{array}{c}\text { Diameter } \\
(\mathrm{mm})\end{array}$ & $\begin{array}{c}\text { Thickness } \\
(\mathrm{mm})\end{array}$ & $\begin{array}{c}\text { Biot number } \\
\text { at } 1000^{\circ} \mathrm{C}\end{array}$ & Sa $(\mu \mathrm{m})$ & Sq $(\mu \mathrm{m})$ & $\begin{array}{c}\text { Ra }(\mu \mathrm{m}) \\
\text { Filter } .8 \mathrm{~mm}\end{array}$ & $\begin{array}{c}\text { Rq }(\mu \mathrm{m}) \\
\text { Filter } .8 \mathrm{~mm}\end{array}$ \\
\cline { 2 - 9 } Sample A & 10 & 1.5 & 0.0003 & 1.09 & 1.38 & 0.253 & 0.412 \\
\hline Sample B & 10 & 2.5 & 0.0004 & 2.10 & 3.03 & 1.08 & 1.41 \\
\hline
\end{tabular}

- Experimental set-up

Emissivity measurement is done in three phases: NIR BI with the spectrometer described in part3, MIR BII with SC7000 FLIR camera and MIR BIII with SC325 FLIR camera. Platinum samples temperature is measured by a $0.3 \mathrm{~mm}$ diameter $\mathrm{K}$ type welded thermocouple protected with ceramic rings, which is also used for temperature regulation. For NIR BI measurements, optical fiber position is held by a fiber holder to ensure repeatability of the measurement. Optical fiber head position is set at $2.5 \mathrm{~mm}$ of the sample surface. The Ag mirror used for BII and BIII measurement exhibit an average reflectance of 0.95 over $[1 \mu \mathrm{m}-14 \mu \mathrm{m}]$. A stainless steel pipe is inserted once the sample temperature is stable in order to assess and remove parasitic signals (see section 3.3.2).
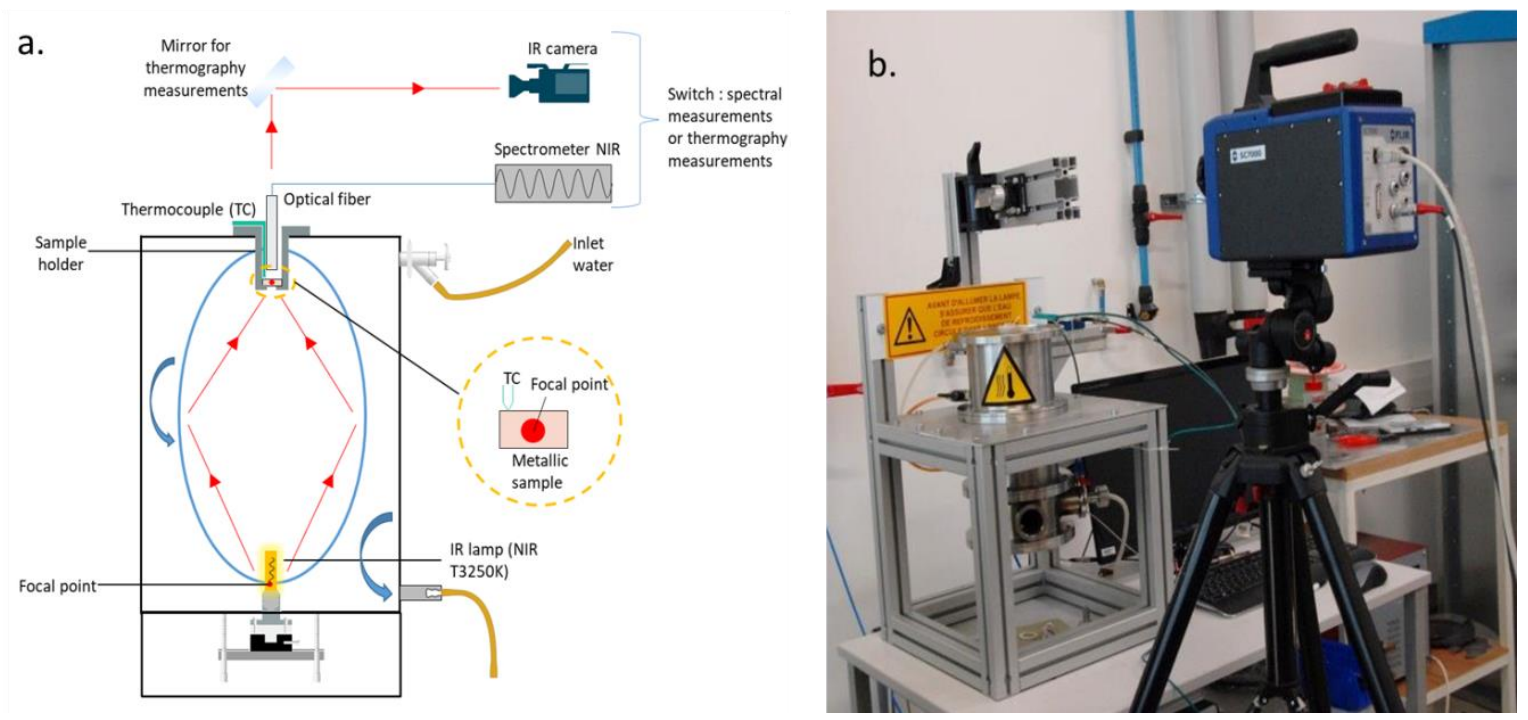

Figure 13: Experimental set-up for emissivity measurement: cross-section view (a), photograph (b)

\subsection{Spectral emissivity measurement \\ - NIR BI measurements}

Platinum spectral emissivity in the NIR range is presented in Figure 14a. Low dependence of spectral emissivity with temperature can be seen which is confirmed by several works ([34], [48]). Spectral emissivity seems to follow two different trends: up to $1.8 \mu \mathrm{m}$ its value is quasi-constant then a regular decrease is observed afterward. The obtained spectra are compared to a measurement performed at $25^{\circ} \mathrm{C}$ using spectrometer Bruker vertex 70 (Figure 15) through an integrating sphere and an InGaAs photodetector (Figure 14b.). The emissivity error in this spectral range can then be estimated computing the relative error between the measurement performed on the apparatus and the one performed by the reference spectrometer at room temperature. 
Except for the measurement at $600^{\circ} \mathrm{C}$, the calculated errors remain low in this spectral range. Error is decreasing when temperatures increases which can be explained by a more favorable signal-to-noise ratio at high temperature. Alike spectral emissivity, error seems to follow two trends: until $2 \mu \mathrm{m}$ its value is quasi-constant and lower than $5 \%$ and a regular increase can be observed after this value. This can be explained by the loss of efficiency for the detector InGaAs in this range. Error value is highest at $2.5 \mu \mathrm{m}$ which corresponds to the end of the spectrometer's spectral range.
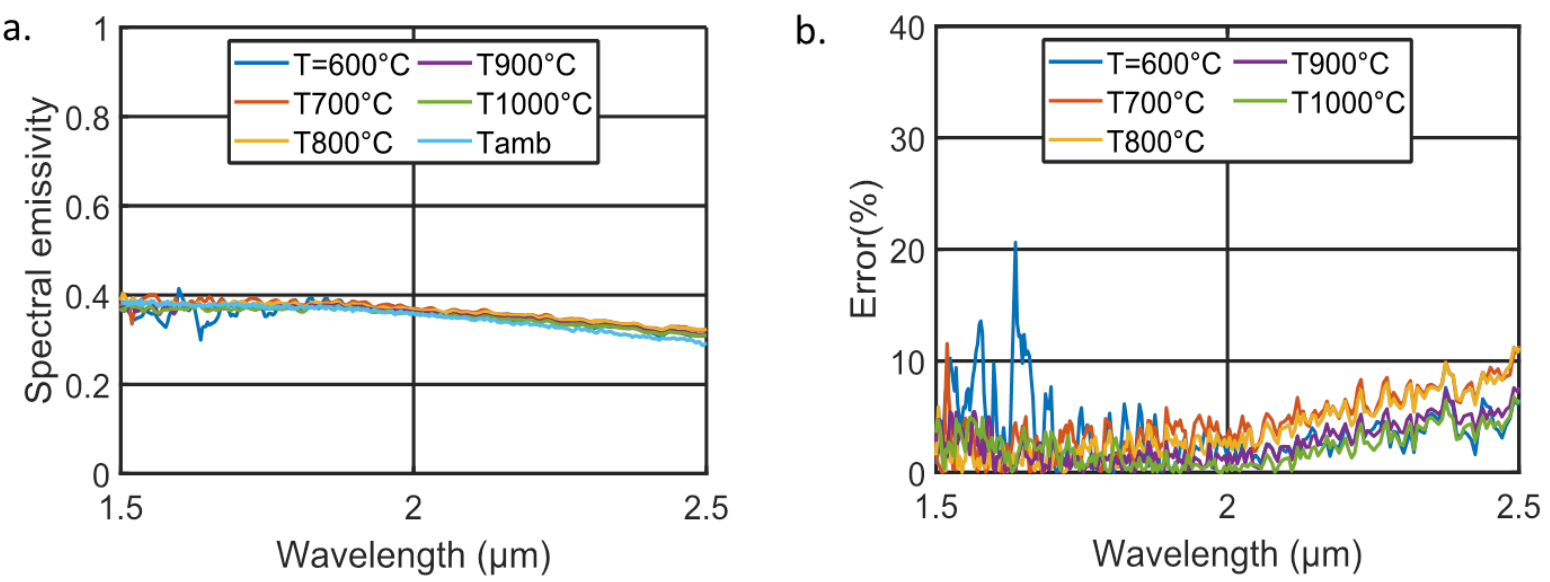

Figure 14 : NIR platinum spectral emissivity (a), relative error compared to reference measurement

(b)

○ MIR BII and BIII measurements

Platinum spectral emissivity values in the MIR BII and BIII bands are presented in Table 8. Unlike measurements in NIR BI, measurements in BII are done for specific wavelengths corresponding to camera's filters described in part 3.1. Alike in the NIR range, emissivity presents a low variation with temperature. Those values are compared to a measurement done through the emission adapter of spectrometer Bruker Vertex 70 equipped with a DLaTGS detector at $400^{\circ} \mathrm{C}$.

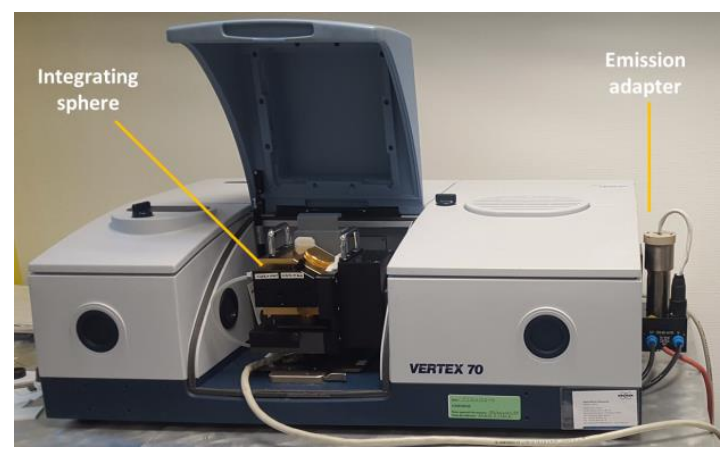

Figure 15 : IRTF spectrometer Bruker Vertex 70 with integrating sphere and emission adapter equipped

Emissivity values measured on the MIR BII are very close to the reference value except for emissivity value at $5.071 \mu \mathrm{m}$ which present a shift between $700^{\circ} \mathrm{C}$ and $1000^{\circ} \mathrm{C}$ but the low value of emissivity at this wavelength induces a high error on measurement. Emissivity measurements on the MIR BIII 
show a higher discrepancy with reference measurement mainly due a low emissivity in such bandwidth and a much higher influence of environment spurious signal.

\begin{tabular}{|c|c|c|c|c|c|}
\hline & \multirow{2}{*}{\multicolumn{4}{|c|}{ Wavelength $(\mu \mathrm{m})$}} & \multirow[b]{4}{*}{ Method } \\
\hline & & & & & \\
\hline & 3.027 & 4 & 5.071 & 10 & \\
\hline Temperature $\left({ }^{\circ} \mathrm{C}\right)$ & \multicolumn{4}{|c|}{ Emissivity } & \\
\hline 400 & 0.17 & 0.13 & 0.11 & 0.08 & spectrometry \\
\hline 600 & 0.16 & 0.14 & 0.10 & 0.12 & \multirow{5}{*}{ This paper } \\
\hline 700 & 0.19 & 0.13 & 0.06 & 0.13 & \\
\hline 800 & 0.18 & 0.14 & 0.07 & 0.16 & \\
\hline 900 & 0.16 & 0.12 & 0.06 & 0.13 & \\
\hline 1000 & 0.16 & 0.13 & 0.07 & 0.12 & \\
\hline
\end{tabular}

Table 8 : MIR platinum spectral emissivity measurements and comparison with a reference measurement

Those values added to spectral emissivities measured in NIR BI are illustrated in Figure 16.

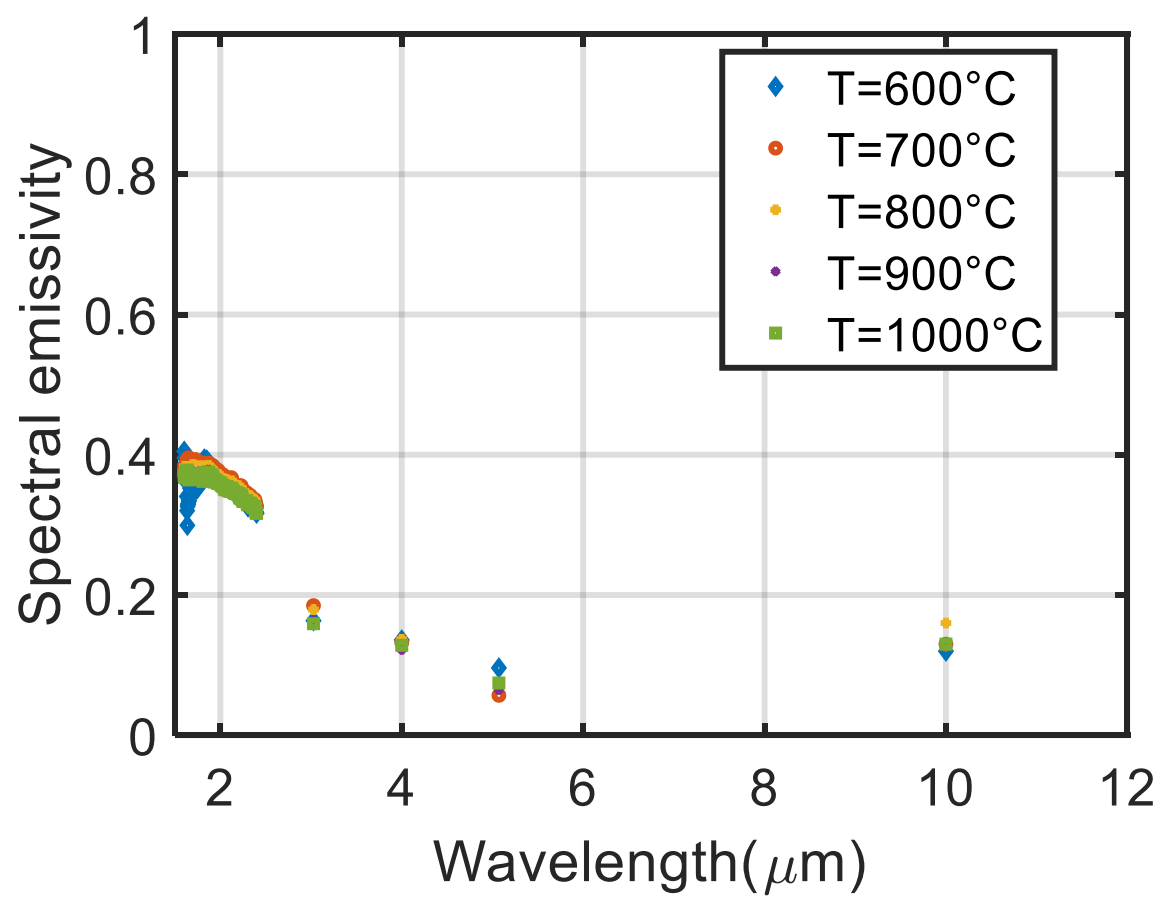

Figure 16: Platinum spectral emissivity measured over NIR and MIR range

The overview of the discontinuous spectrum over a wide spectral range indicates that these variations are typical of metallic materials, with a monotonous low decrease of emissivity with wavelength. Moreover, the discontinuous values in $\mathrm{BI}$ and $\mathrm{BII}$ seem to be spectrally linked, which reinforces the physical consistency of the measurement. The value at $10 \mu \mathrm{m}$ is over-estimated, but it will be showed in next part that this error is not critical for total emissivity calculation. 


\subsection{Integrated emissivity computation}

As discussed in section in section 3.2., the integrated emissivity can be approximated from piecewise measurements through the use of interpolation. This interpolated emissivity is then integrated as follows.

$$
\varepsilon(T)=\frac{\int_{\lambda_{1}}^{\lambda_{2}} \varepsilon_{\lambda}(T) * L^{0}(\lambda, T) * d \lambda}{\int_{\lambda_{1}}^{\lambda_{2}} L^{0}(\lambda, T) * d \lambda}
$$

Blackbody radiance and interpolated platinum radiance corresponding to blackbody radiance multiplied by platinum interpolated emissivity are presented on Figure 17. Curves present similar shapes for both cases with a similar evolution with temperature. Radiance value at $10 \mu \mathrm{m}$ is very low as compared to NIR BI and MIR BII corresponding values which confirms that the measurement error will have a low impact on integrated emissivity value.

a.

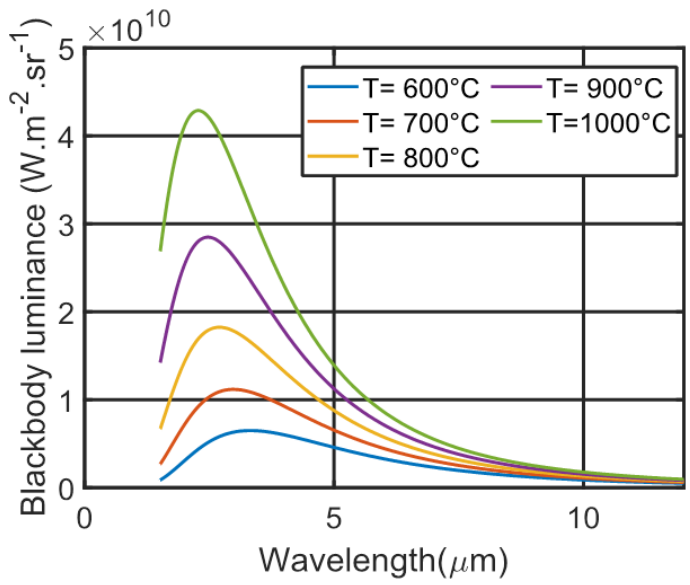

b.

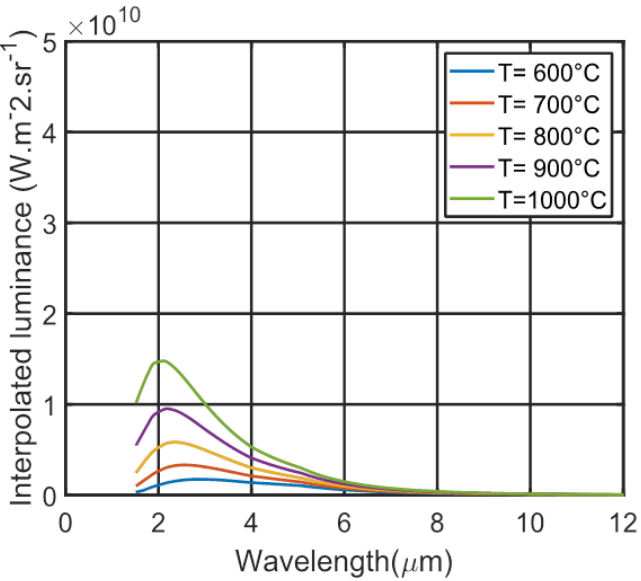

Figure 17 : Blackbody radiance (a), Platinum interpolated radiance (b)

Those values are integrated between 1.3 and $11 \mu \mathrm{m}$ in order to provide integrated emissivity. The obtained results are summarized in Table 9 and depicted in Figure 18. It is seen that integrated emissivity monotonically increases with temperature which is acknowledged as classical evolution for metallic materials [1]. Moreover, those results are very close to the integrated emissivity (reference) calculated with the spectrometer Bruker Vertex 70. This extrapolation with temperature is appropriate in this case considering that Platinum do not oxidize with temperature.

\begin{tabular}{|c|c|c|c|c|c|}
\hline Temperature $\left({ }^{\circ} \mathrm{C}\right)$ & 600 & 700 & 800 & 900 & 1000 \\
\hline Integrated emissivity & 0.13 & 0.14 & 0.16 & 0.17 & 0.19 \\
\hline $\begin{array}{c}\text { Reference } \\
\text { integrated emissivity }\end{array}$ & 0.15 & 0.16 & 0.18 & 0.19 & 0.20 \\
\hline
\end{tabular}

Table 9 : Platinum integrated emissivity at various temperatures

Finally, the integrated emissivities calculated in this work are compared to total emissivity values found in literature [49] [50] [51] [52] [53]. Compared values are in the same order of magnitude and present a similar evolution with temperature. The offset visible in Figure 18 can be explained by the 
difference of roughness between the platinum samples and the differences in measurement methodology.

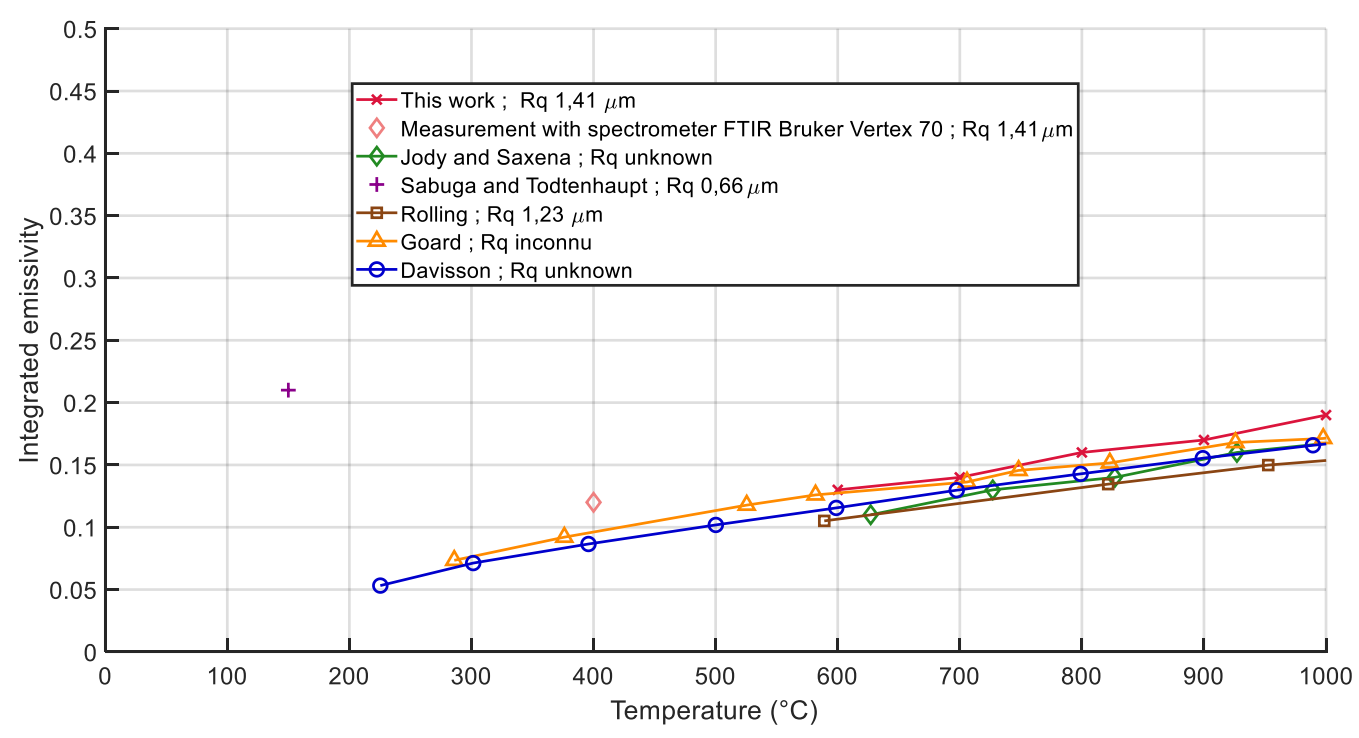

Figure 18: Integrated emissivity of platinum compared with literature data

The values measured and calculated form spectral data in this paper are very consistent with previous work available in the literature. It shows a global increase of total emissivity with temperature, which is, one again, very typical of metallic materials.

\section{Conclusion}

In this paper, a new apparatus for both spectral and total emissivity measurement at high temperatures (typ. $1000^{\circ} \mathrm{C}$ ) is described. The constraints at such temperatures have required a rigorous selection of hardware: lamps, sample holder, measuring devices. In addition, a ray tracing model has been developing in order to address the sample dimension and positioning issues.

The selection of adequate sensors that enable the measurement of integrated emissivity between $600^{\circ} \mathrm{C}$ and $1000^{\circ} \mathrm{C}$, is extensively discussed. For the NIR band, a spectrometer operating between 1.3 and $2.5 \mu \mathrm{m}$ is selected. For the MIR bands (BII and BIII), cameras equipped with optical filters were selected to measure spectral emissivity at 3.027, 4, 5.071 and $10 \mu \mathrm{m}$. An interpolation strategy has been adopted to minimize the discontinuity of the spectral bands on the value of total emissivity. All the sensors have been calibrated with a blackbody. A detailed methodology for environmental parasitic signals removal is proposed. A reference material is then used to validate the measurement device. The choice was guided towards Platinum due its chemical stability, its low oxygen reactivity and low emissivity variation over the NIR band.

NIR measurements confirmed that the emissivity remains constant within this band. A comparison is then made with measurements done by a commercial spectrometer at ambient temperature. The results show a good agreement between values obtained with those two methods. Measurements over the MIR BII also show a good accuracy with reference measurement. Measurements performed in the MIR BIII showed the biggest shift due to a decrease of signal-to-noise ratio. Moreover, high 
reflecting materials like platinum are not a favorable case for emissivity measurement especially in MIR BIII where emissivity value is low. Nevertheless, it has been shown that an error at this wavelength have a low impact on integrated emissivity value for targeted temperatures on this paper. Spectral emissivities were then integrated to give integrated emissivities as a function of temperature. Those calculated values show a good agreement with values found in other published papers despite a discrepancy in term of surface roughness.

The results presented in the present paper validate the use of such device for spectral and integrated emissivity measurement purpose. Moreover, targeted materials for hot forming applications (titanium alloys, nickel alloys...) have higher emissivities and will have a much more favorable signalto-noise ratio.

The crucial originality of this device stands in the heating principle by an elliptical furnace which allows high heating rate, ramp temperatures and temperature bearing with a design allowing to easily switch between different atmospheric conditions. Moreover, this versatility also applies to sensor selection. Indeed, it was shown in this paper the possibility to perform emissivity measurement by both spectrometry and thermography, but those sensors can easily be substituted by other sensors like thermopile or photodiodes. The use of a LVF (Linear Variable Filter) associated with an adequate sensor or a spectrometer with a wider spectral range ranging from NIR BI to MIR BIII are among the most promising perspectives. This versatility on heating system, sensor selection, cooling system and experimental conditions is the main strength of the device.

Sample holder radiation is the main difficulty on this device. Therefore, an adaptation of the sample holder to simplify the spurious signal removing method is also currently studied. A cooling system and an improvement of the screening are considered accordingly. The device has also been adapted to perform emissivity measurements under vacuum or inert atmosphere which can be interesting for spatial applications or some forming processes done under inert atmosphere. The device can also perform measurements for temperature cycles or bearings which can give an emissivity function of heating time and potentially allow linking emissivity with an oxidation thickness. Cost-efficiency and simplicity of the system also allow applying this method for non-specialist laboratories.

Acknowledgements: Authors would like to thank Adrien Lieurey for his great help on this device development. The R\&D project FASTE2, winner of the EASYNOV call for papers, is funded by Occitanie region via the FEDER. The team would like to thank the FASTE2 partners (AUROCK and NEOTIM) for their help and contribution to this project. 
References:

[1] J. R. Howell, R. Siegel, and M. P. Mengüç, Thermal radiation heat transfer. .

[2] L. Ibos, M. Marchetti, A. Boudenne, S. Datcu, Y. Candau, and J. Livet, "Infrared emissivity measurement device: principle and applications," Meas. Sci. Technol., vol. 17, no. 11, pp. 2950-2956, Nov. 2006.

[3] M. Vollmer and K.-P. Möllmann, Infrared thermal imaging: fundamentals, research and applications. .

[4] P. B. Coates, "Multi-Wavelength Pyrometry," Metrologia, vol. 17, no. 3, pp. 103-109, Jul. 1981.

[5] L. González-Fernández, L. del Campo, R. B. Pérez-Sáez, and M. J. Tello, “Normal spectral emittance of Inconel 718 aeronautical alloy coated with yttria stabilized zirconia films," J. Alloys Compd., vol. 513, pp. 101-106, Feb. 2012.

[6] K. Yu, H. Zhang, Y. Liu, and Y. Liu, "Study of normal spectral emissivity of copper during thermal oxidation at different temperatures and heating times," Int. J. Heat Mass Transf., vol. 129, pp. 1066-1074, Feb. 2019.

[7] E. Billur, "Hot formed steels," Automot. Steels, pp. 387-411, Jan. 2017.

[8] G. Bernhart, P. Lours, T. Cutard, V. Velay, and F. Nazaret, "Processes and equipment for superplastic forming of metals," in Superplastic Forming of Advanced Metallic Materials, Elsevier, 2011, pp. 49-71.

[9] H. Karbasian and A. E. Tekkaya, "A review on hot stamping," J. Mater. Process. Technol., vol. 210, no. 15, pp. 2103-2118, Nov. 2010.

[10] D. P. DeWitt and G. D. Nutter, Eds., Theory and Practice of Radiation Thermometry. Hoboken, NJ, USA: John Wiley \& Sons, Inc., 1988.

[11] M. F. (Michael F. . Modest, Radiative heat transfer. Elsevier Science, 2013.

[12] D. G. Goebel, "Generalized Integrating-Sphere Theory," Appl. Opt., vol. 6, no. 1, p. 125, Jan. 1967.

[13] H. Wakabayashi and T. Makino, "A new spectrophotometer system for measuring thermal radiation phenomena in a 0.30-11 $\mu \mathrm{m}$ wavelength region," 2001.

[14] T. Makino and H. Wakabayashi, "A New Spectrophotometer System for Measuring Hemispherical Reflectance and Normal Emittance of Real Surfaces Simultaneously," Int. J. Thermophys., vol. 31, no. 11-12, pp. 2283-2294, Dec. 2010.

[15] J. Hameury, B. Hay, and J. R. Filtz, "Measurement of Infrared Spectral Directional Hemispherical Reflectance and Emissivity at BNM-LNE," Int. J. Thermophys., vol. 26, no. 6, pp. 1973-1983, Nov. 2005.

[16] Rémi Gilblas, "Mesure de champs de températures vraies par thermo-réflectométrie proche infrarouge," 2012.

[17] R. Gilblas, T. Sentenac, D. Hernandez, and Y. Le Maoult, "Thermoreflectometry: a new system to determine the true temperature fields on surface with unknown emissivities," 2011, p. 801305. 
[18] T. Fu, M. Duan, J. Tang, and C. Shi, "Measurements of the directional spectral emissivity based on a radiation heating source with alternating spectral distributions," Int. J. Heat Mass Transf., vol. 90, pp. 1207-1213, Nov. 2015.

[19] B. T. Barnes, W. E. Forsythe, and E. Q. Adams, "The Total Emissivity of Various Materials at 100-500'," J. Opt. Soc. Am., vol. 37, no. 10, p. 804, Oct. 1947.

[20] M. Rink, V. Kromchenko, S. Mekhontsev, and L. M. Hanssen, "Near Infrared Indirect Emissivity Measurements and System Employing a Vacuum Goniometer | NIST." 25-Jun-2004.

[21] T. J. Warren, N. E. Bowles, K. Donaldson Hanna, and I. R. Thomas, "The Oxford space environment goniometer: A new experimental setup for making directional emissivity measurements under a simulated space environment," Rev. Sci. Instrum., vol. 88, no. 12, p. 124502, Dec. 2017.

[22] M. Honner and P. Honnerová, "Survey of emissivity measurement by radiometric methods," Appl. Opt., vol. 54, no. 4, p. 669, Feb. 2015.

[23] S. Clausen, A. Morgenstjerne, and O. Rathmann, "Measurement of surface temperature and emissivity by a multitemperature method for Fourier-transform infrared spectrometers," Appl. Opt., vol. 35, no. 28, p. 5683, Oct. 1996.

[24] R. M. Sova, M. J. Linevsky, M. E. Thomas, and F. F. Mark, "High-temperature infrared properties of sapphire, AION, fused silica, yttria, and spinel," Infrared Phys. Technol., vol. 39, no. 4, pp. 251-261, Jun. 1998.

[25] L. del Campo, R. B. Pérez-Sáez, X. Esquisabel, I. Fernández, and M. J. Tello, “New experimental device for infrared spectral directional emissivity measurements in a controlled environment," Rev. Sci. Instrum., vol. 77, no. 11, p. 113111, Nov. 2006.

[26] B. Hay et al., "New Facilities for the Measurements of High-Temperature Thermophysical Properties at LNE," Int. J. Thermophys., vol. 35, no. 9-10, pp. 1712-1724, Oct. 2014.

[27] D. Demange, M. Bejet, and B. Dufour, "New Methods for Measuring the Thermal Emissivity of Semi-transparent and Opaque Materials," in Proceedings of the 2006 International Conference on Quantitative InfraRed Thermography, 2006.

[28] J. Ishii and A. Ono, "Uncertainty estimation for emissivity measurements near room temperature with a Fourier transform spectrometer," Meas. Sci. Technol., vol. 12, no. 12, pp. 2103-2112, Dec. 2001.

[29] J. Hameury, "Determination of uncertainties for emissivity measurements in the temperature range $200-800^{\circ} \mathrm{C}$," High Temp. Press., vol. 30, no. 2, pp. 223-228, 1998.

[30] A. Pantinakis, N. K.-I. journal of thermophysics, and undefined 2001, "A simple highsensitivity radiometer in the infrared for measurements of the directional total emissivity of opaque materials at near-ambient temperatures," Springer.

[31] V. Dozhdikov, V. P.-H. T. H. Pressures, and undefined 1995, "New automated apparatus for the measurement of spectral emissivity of nonconducting materials by high-speed spectrometer," Pion.

[32] D. Hernandez, J. L. Sans, A. Netchaieff, P. Ridoux, and V. Le Sant, "Experimental validation of a pyroreflectometric method to determine the true temperature on opaque surface without hampering reflections," Measurement, vol. 42, no. 6, pp. 836-843, Jul. 2009. 
[33] F. Zhang, K. Yu, K. Zhang, Y. Liu, K. Xu, and Y. Liu, "An emissivity measurement apparatus for near infrared spectrum," Infrared Phys. Technol., vol. 73, pp. 275-280, 2015.

[34] D. De Sousa Meneses, P. Melin, L. del Campo, L. Cosson, and P. Echegut, "Apparatus for measuring the emittance of materials from far infrared to visible wavelengths in extreme conditions of temperature," Infrared Phys. Technol., vol. 69, pp. 96-101, Mar. 2015.

[35] M. Balat-Pichelin, J. F. Robert, and J. L. Sans, "Emissivity measurements on carbon-carbon composites at high temperature under high vacuum," Appl. Surf. Sci., vol. 253, no. 2, pp. 778783, Nov. 2006.

[36] M. Balat-Pichelin and A. Bousquet, "Total hemispherical emissivity of sintered SiC up to 1850 $\mathrm{K}$ in high vacuum and in air at different pressures," J. Eur. Ceram. Soc., vol. 38, no. 10, pp. 3447-3456, Aug. 2018.

[37] R. N. Rogers and E. D. Morris, "Determination of Emissivities with a Differential Scanning Calorimeter.," Anal. Chem., vol. 38, no. 3, pp. 410-412, Mar. 1966.

[38] S. Sasaki, H. Masuda, M. Higano, and N. Hishinuma, "Simultaneous measurements of specific heat and total hemispherical emissivity of chromel and alumel by a transient calorimetric technique," Int. J. Thermophys., vol. 15, no. 3, pp. 547-565, May 1994.

[39] G. . Greene, C. . Finfrock, and T. . Irvine, "Total hemispherical emissivity of oxidized Inconel 718 in the temperature range $300-1000^{\circ}$ C," Exp. Therm. Fluid Sci., vol. 22, no. 3-4, pp. 145153, Sep. 2000.

[40] S. Moghaddam, J. Lawler, J. Currano, and J. Kim, "Novel Method for Measurement of Total Hemispherical Emissivity," J. Thermophys. Heat Transf., vol. 21, no. 1, pp. 128-133, Jan. 2007.

[41] T. Fu and P. Tan, "Transient Calorimetric Measurement Method for Total Hemispherical Emissivity," J. Heat Transfer, vol. 134, no. 11, p. 111601, Nov. 2012.

[42] V. Pozzobon, S. Salvador, J. Bézian, ... M. E.-H.-F. P., and undefined 2014, "Radiative pyrolysis of wet wood under intermediate heat flux: Experiments and modelling," Elsevier.

[43] B. Cosson, F. Schmidt, Y. Le Maoult, and M. Bordival, "Infrared heating stage simulation of semi-transparent media (PET) using ray tracing method," Int. J. Mater. Form., vol. 4, no. 1, pp. 1-10, Mar. 2011.

[44] W. H. (William H. McAdams and National Research Council (U.S.). Committee on Heat Transmission., Heat transmission. R.E. Krieger Pub. Co, 1985.

[45] E. D. PALIK, "Introductory Remarks," in Handbook of Optical Constants of Solids, Elsevier, 1985, pp. 3-9.

[46] H. Akima and Hiroshi, "A New Method of Interpolation and Smooth Curve Fitting Based on Local Procedures," J. ACM, vol. 17, no. 4, pp. 589-602, Oct. 1970.

[47] H. Watanabe, J. Ishii, H. Wakabayashi, T. Kumano, and L. Hanssen, "Spectral Emissivity Measurements," in Experimental Methods in the Physical Sciences, vol. 46, 2014, pp. 333-366.

[48] S. Deemyad and I. F. Silvera, "Temperature dependence of the emissivity of platinum in the IR," Rev. Sci. Instrum., vol. 79, no. 8, p. 086105, Aug. 2008.

[49] B. J. Jody and S. C. Saxena, "Radiative heat transfer from metal wires: hemispherical total emittance of platinum," J. Phys. E., vol. 9, no. 5, pp. 359-362, May 1976. 
[50] W. Sabuga and R. Todtenhaupt, "Effect of roughness on the emissivity of the precious metals silver, gold, palladium, platinum, rhodium, and iridium," High Temp. Press., vol. 33, no. 3, pp. 261-269, 2001.

[51]; R E Rolling and A. I. Funai, "INVESTIGATION OF THE EFFECT OF SURFACE CONDITIONS "ON THE RADIANT PROPERTIES OF METALS Part II, Measurements on Roughened Platintm and Oxidized Stainless Steel," 1967.

[52] P. R. C. Goard, "Application of hemispherical surface pyrometers to the measurement of the emissivity of platinum (a low-emissivity material)," J. Sci. Instrum., vol. 43, no. 4, pp. 256-258, Apr. 1966.

[53] C. Davisson and J. R. Weeks, "The Relation between the Total Thermal Emissive Power of a Metal and its Electrical Resistivity," J. Opt. Soc. Am., vol. 8, no. 5, p. 581, May 1924. 
a.

Controller b.

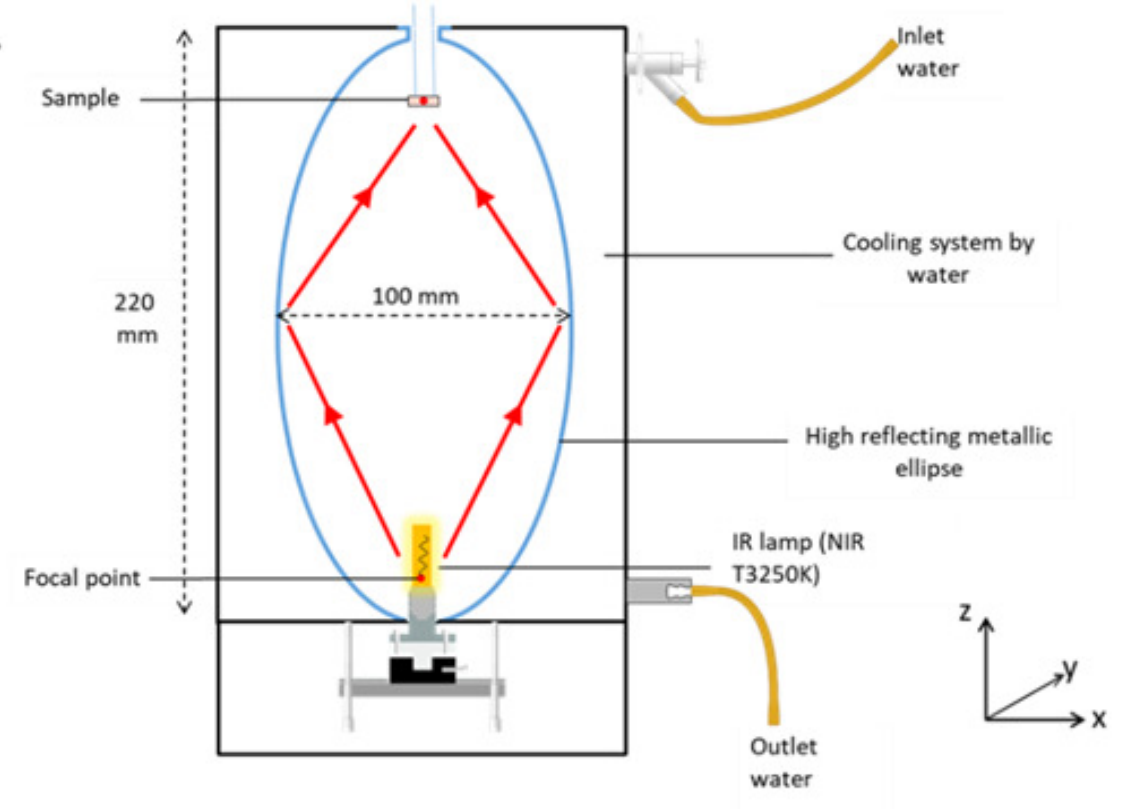


Sample Holder

Sample

Ellipse

Coolant fluid

Bulb

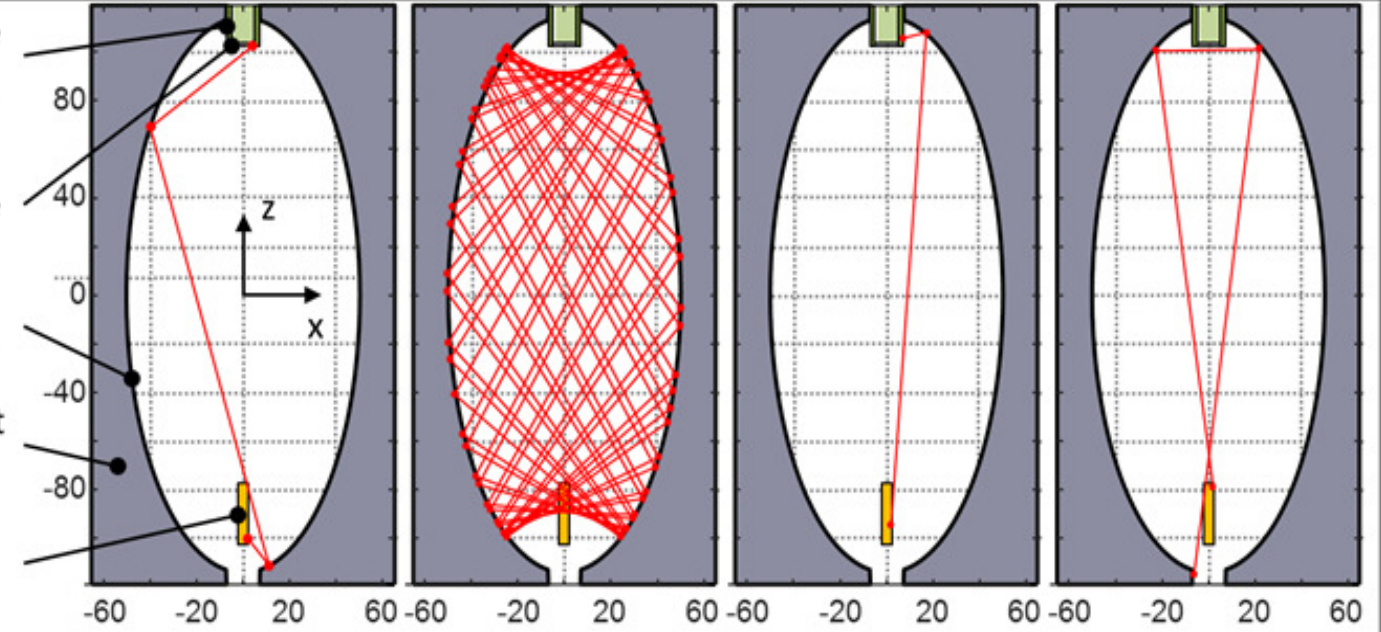

a) The ray hits the sample

b) Too many reflections c) The ray hits the sample holder d) The ray hits bulb base

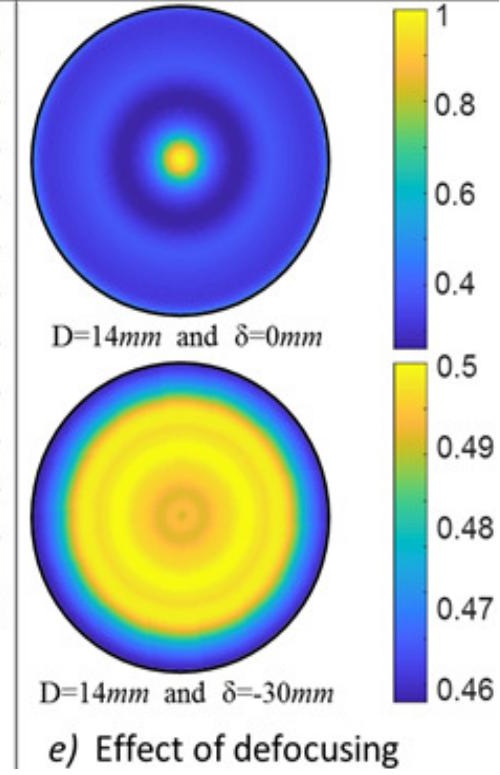


a.

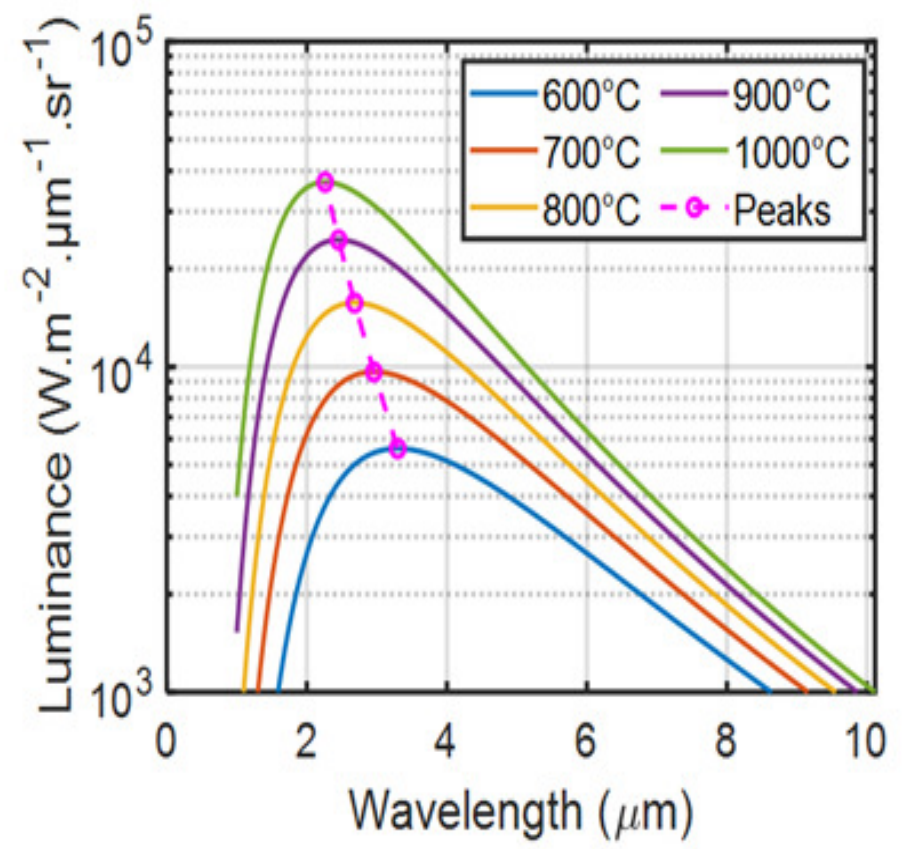

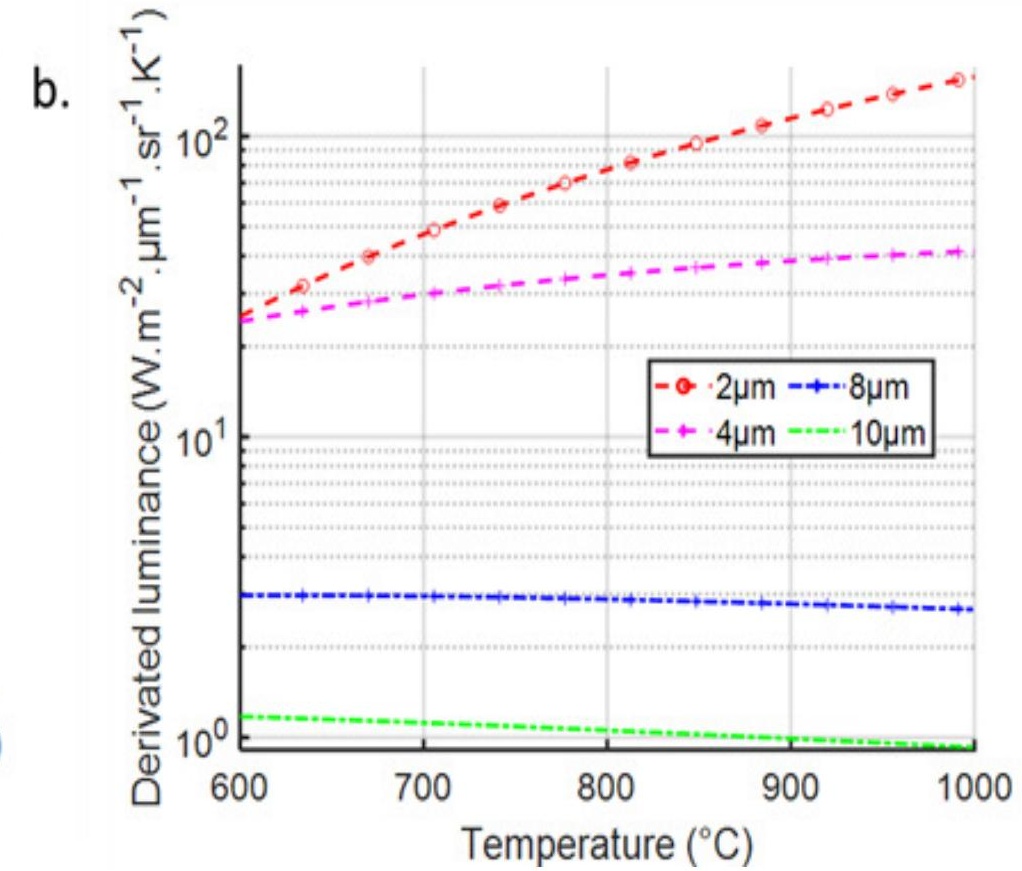




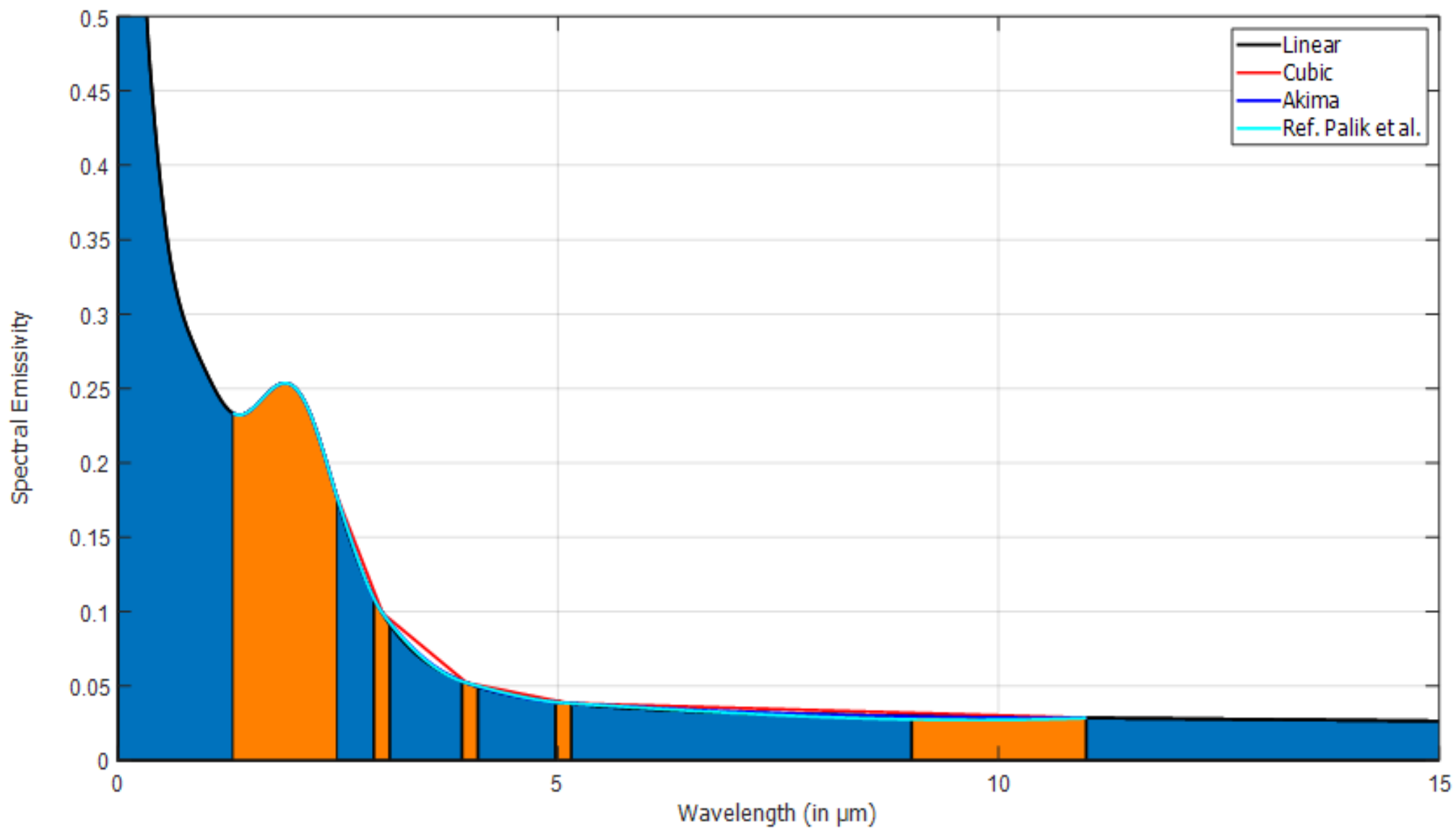




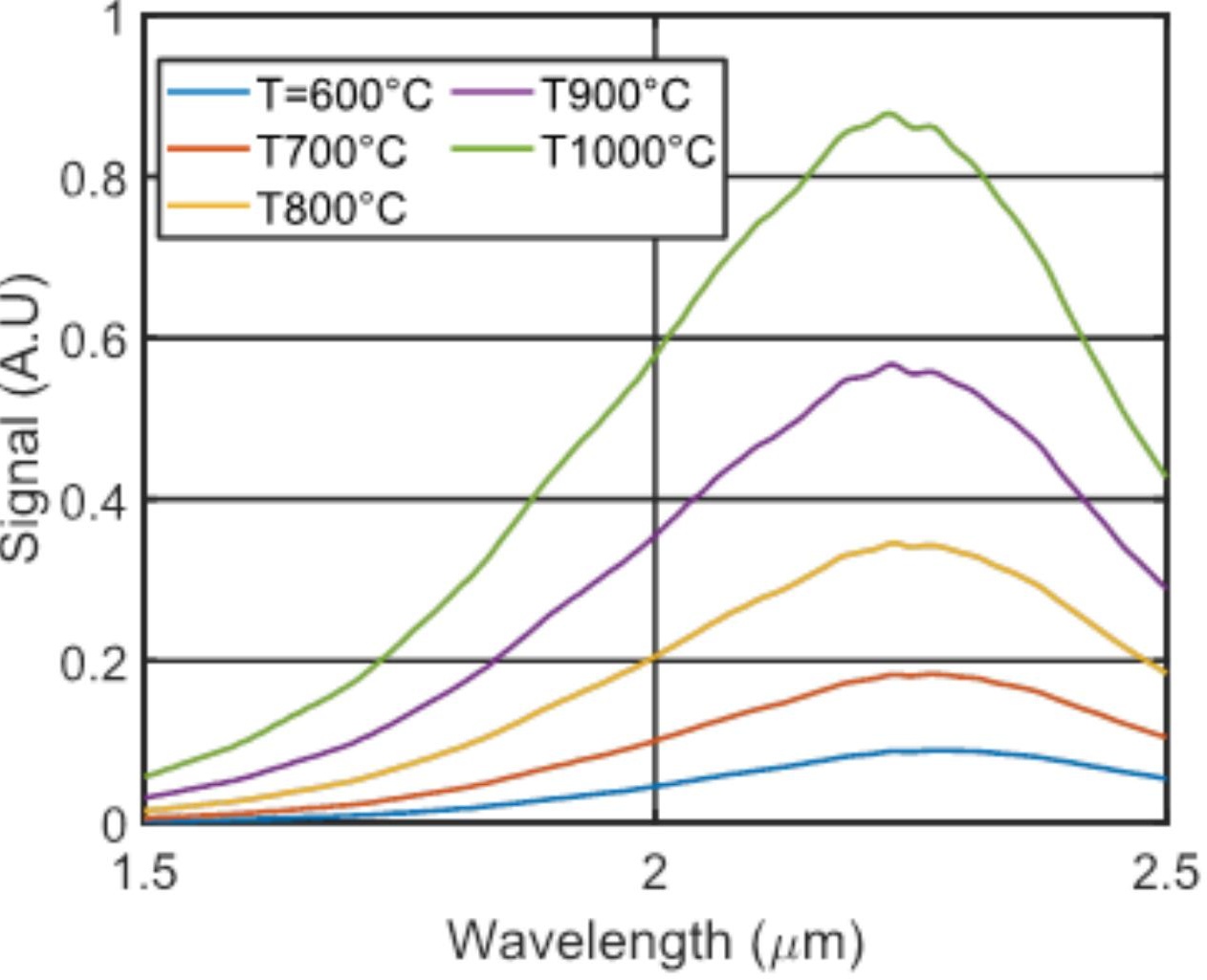


a.
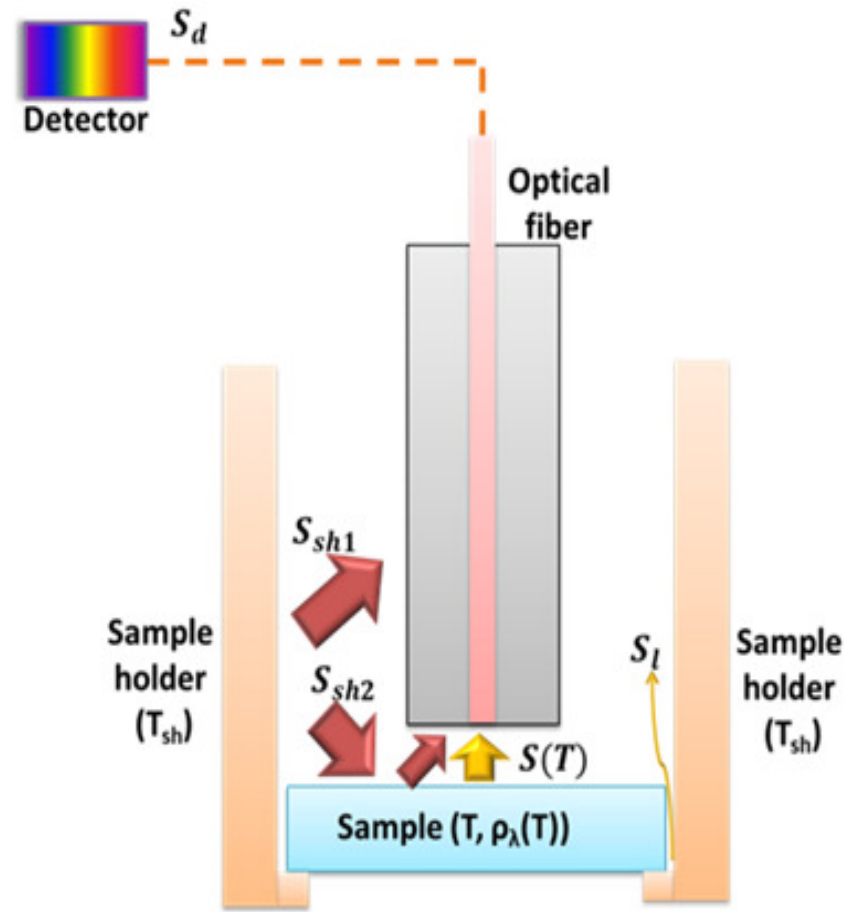

\section{Ag mirror}

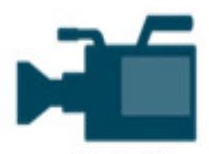

Detector

$S_{d}$
Sample

holder

$\left(\mathrm{T}_{\mathrm{sh}}\right)$ holder

$\left(T_{\text {sh }}\right)$

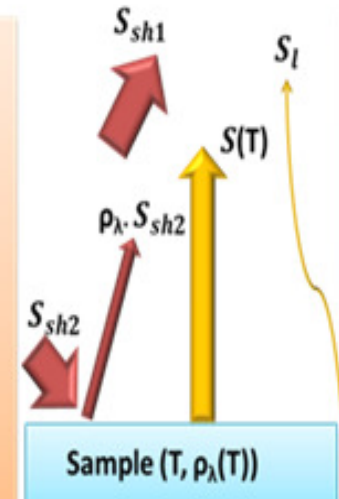


a.

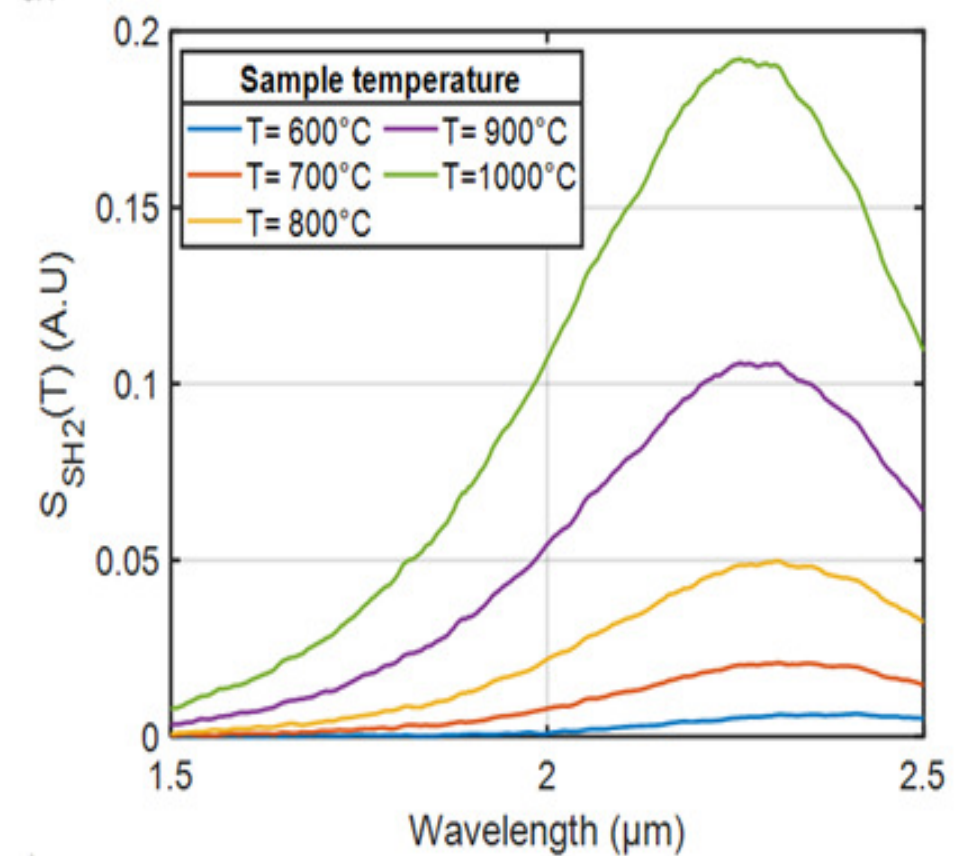

b.

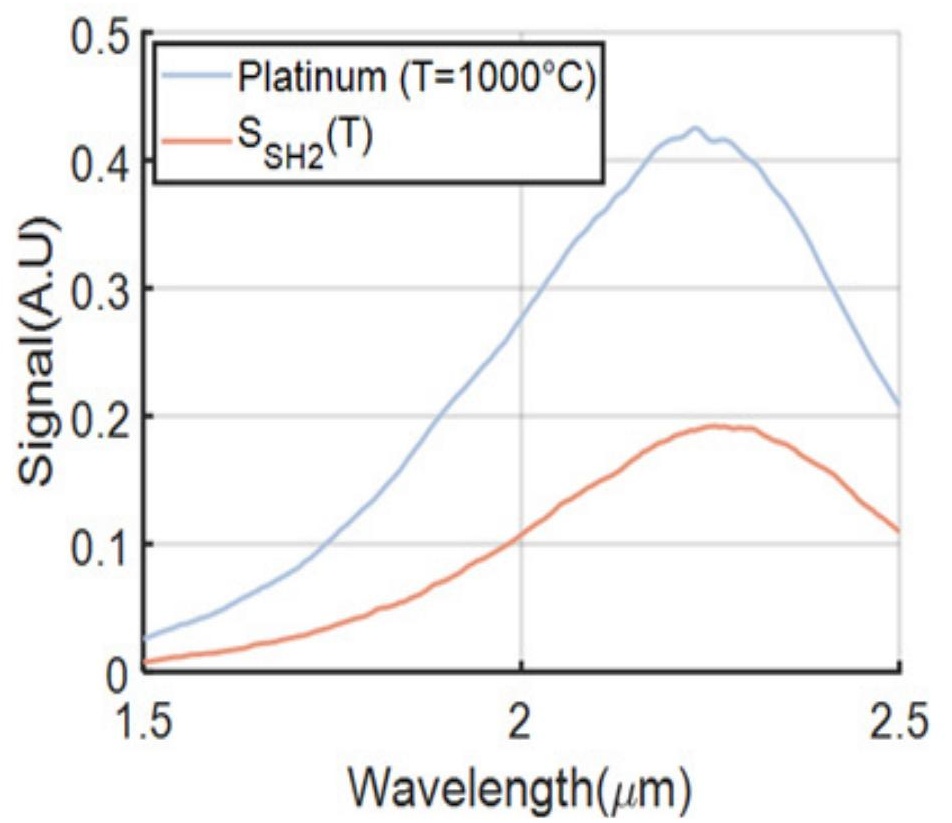



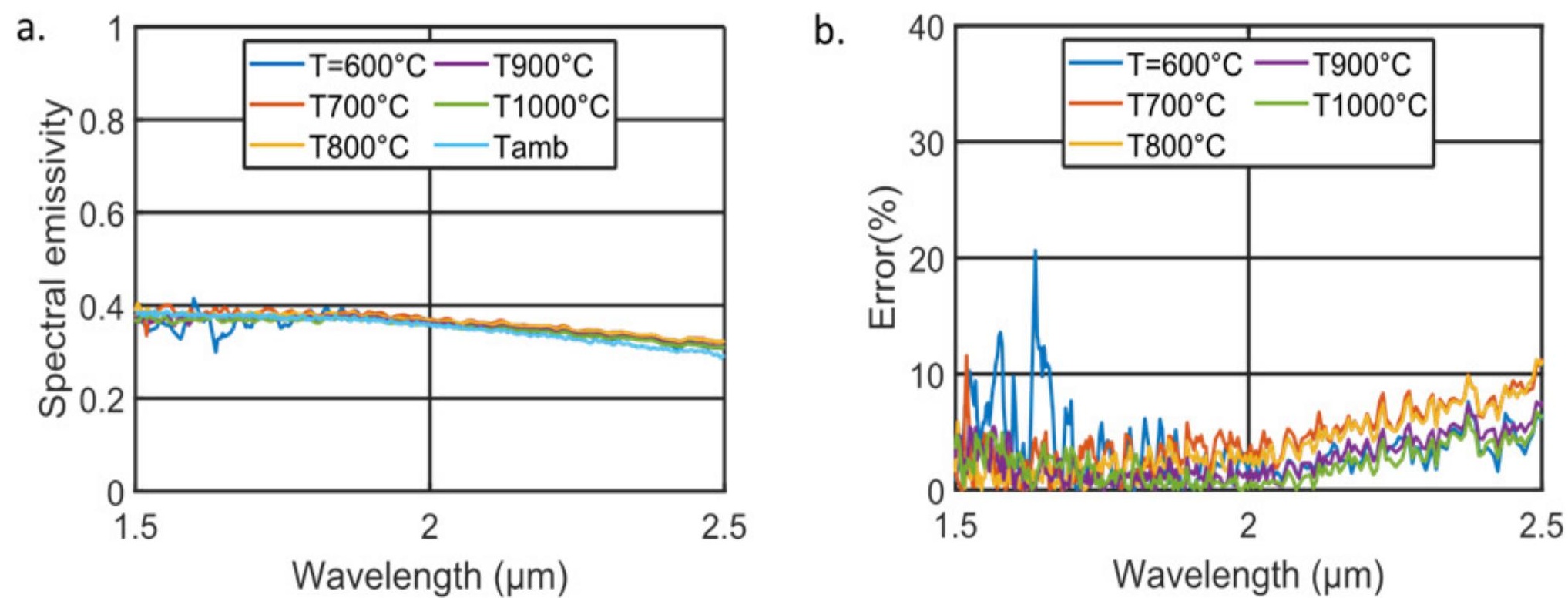

Wavelength $(\mu \mathrm{m})$ 


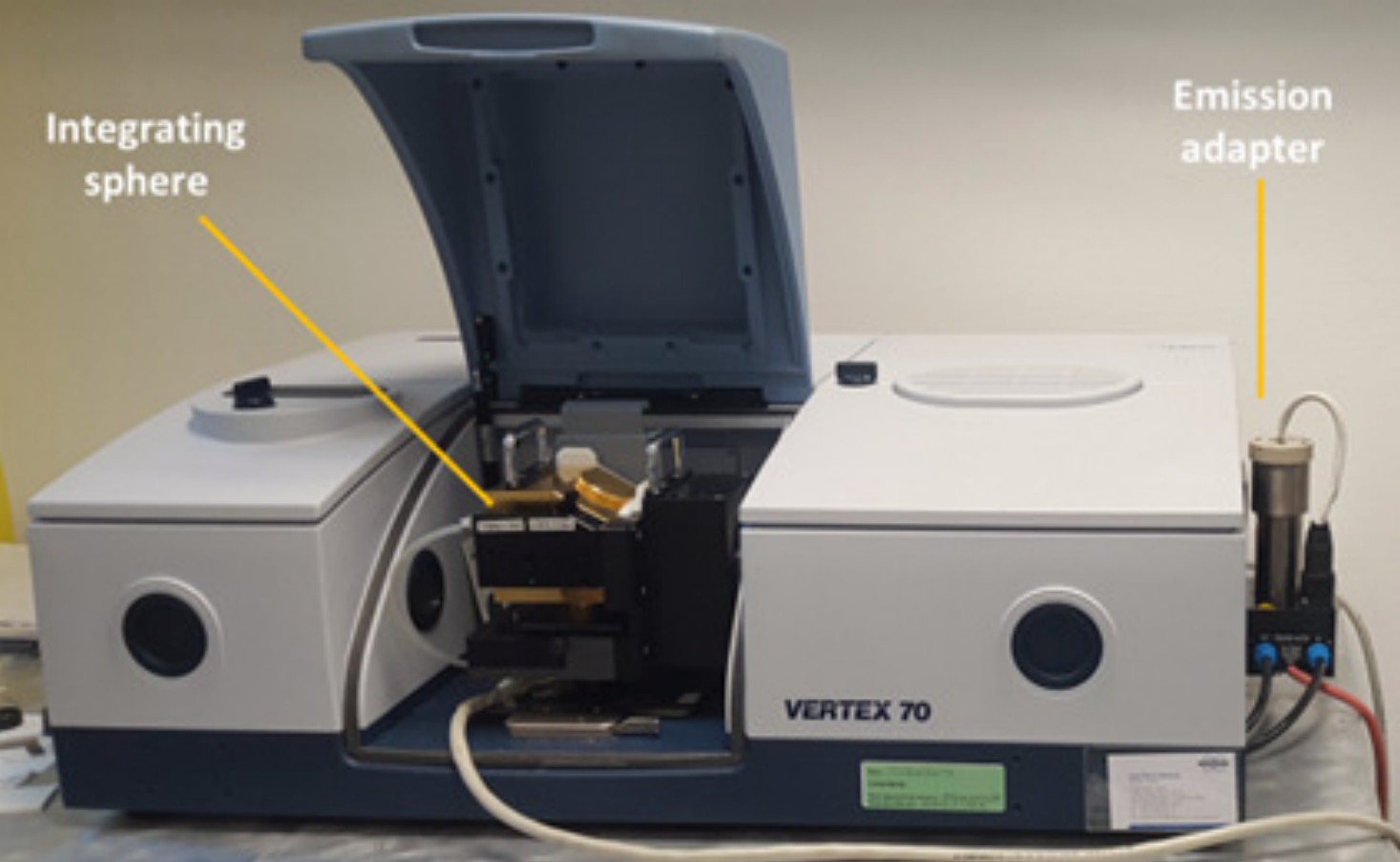


a.

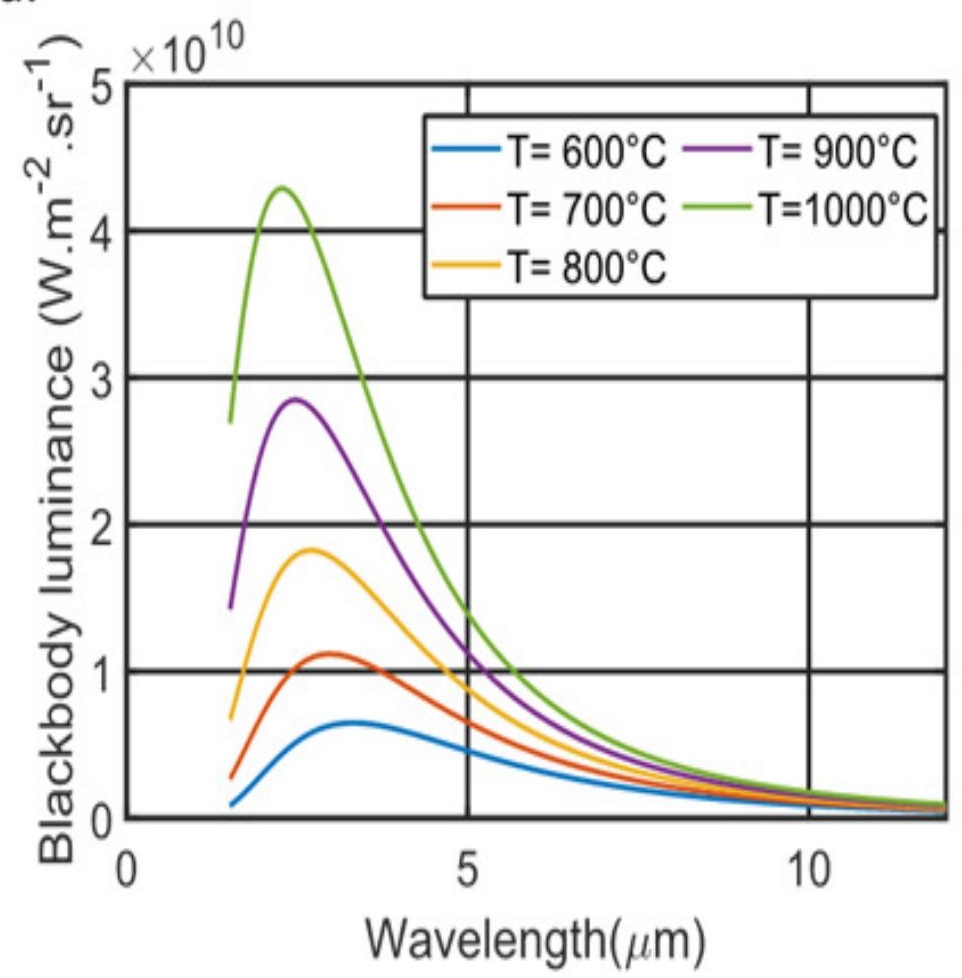

b.

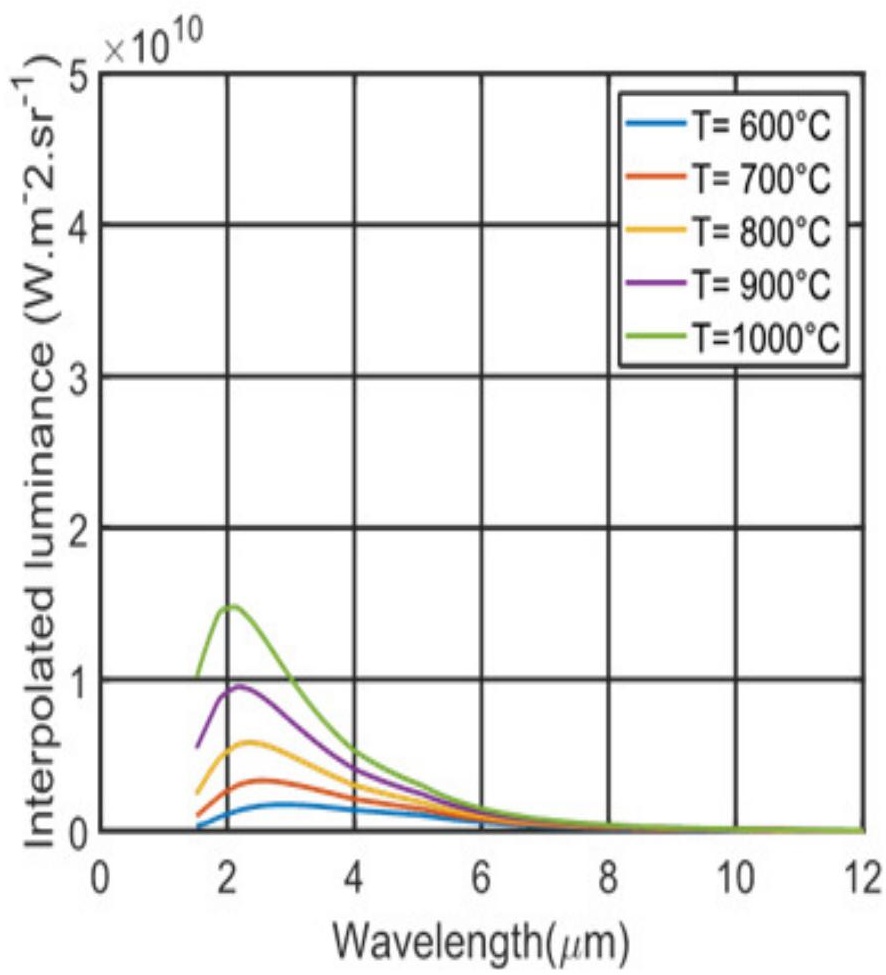




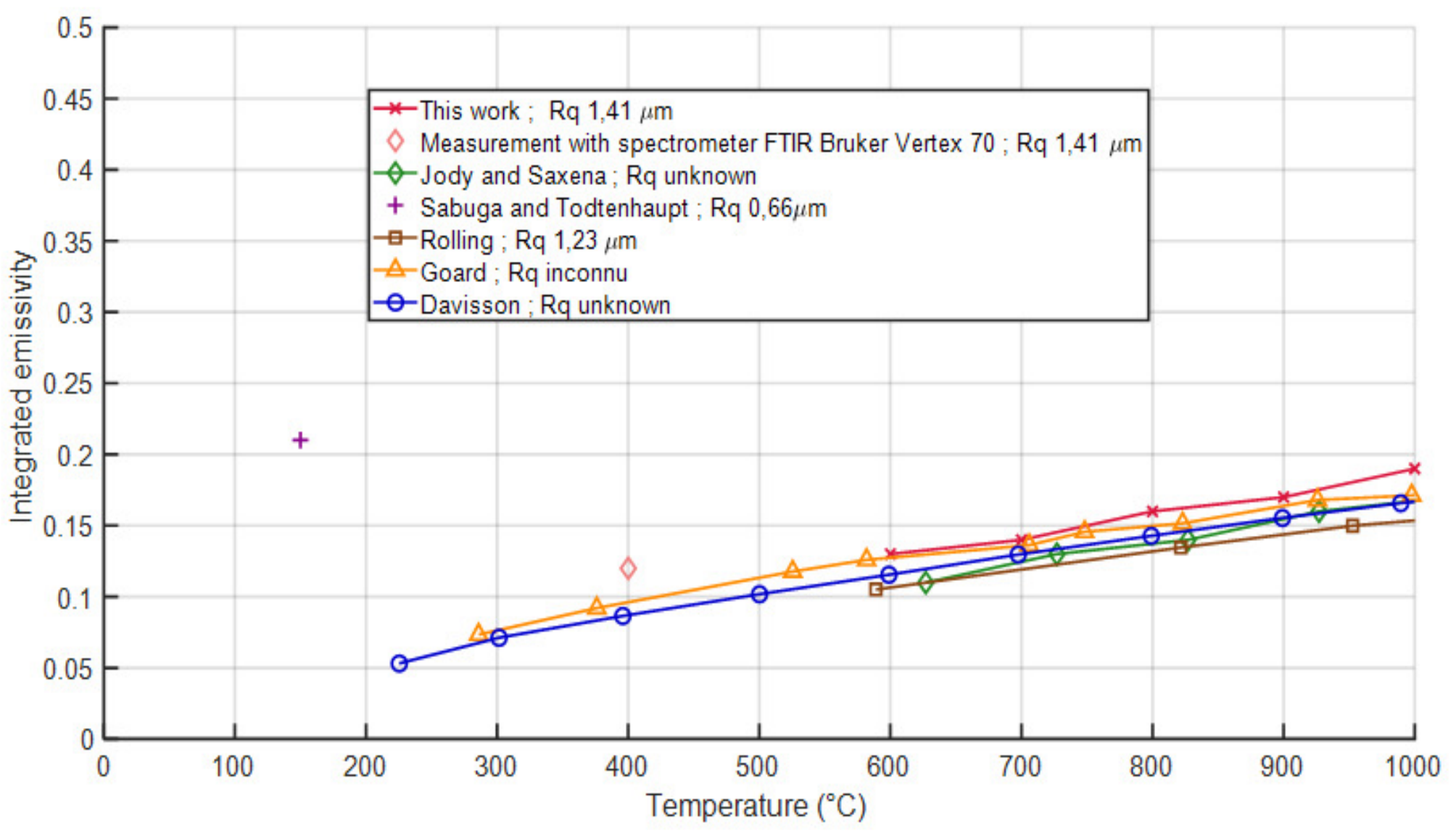

